\title{
Genome-wide characterization and expression analysis of PP2CA family members in response to ABA and osmotic stress in Gossypium
}

\author{
Tingting Lu ${ }^{\text {Equal first author, } 1,2}$, Gaofeng Zhang ${ }^{\text {Equal first author, } 1}{ }^{,}$Yibin Wang ${ }^{1}$, Shibin He ${ }^{1}$, Lirong Sun ${ }^{\text {Corresp., } 1}{ }^{\text {, Fushun Hao }}{ }^{\text {Corresp. }}$ \\ ${ }^{1}$ State Key Laboratory of Cotton Biology, Henan Key Laboratory of Plant Stress Biology, School of Life Sciences, Henan University, Kaifeng, Henan, China \\ 2 Henan University of Animal Husbandry and Economy, Zhengzhou, Henan, China \\ Corresponding Authors: Lirong Sun, Fushun Hao \\ Email address: sunlr9208@henu.edu.cn, haofsh@henu.edu.cn
}

Clade A type 2C protein phosphatases (PP2CAs), as central regulators of abscisic acid (ABA) signaling, negative control growth, development and responses to multiple stresses in plants. PP2CA gene families have been characterized at genome-wide levels in several diploid plants like Arabidopsis and rice. However, the information about genome organization, phylogenesis and putative functions of PP2CAs in Gossypium is lacking. Here, PP2CA family members were comprehensively analyzed in four Gossypium species including the diploid progenitor $G$. arboretum, G. raimondii and the tetraploid $G$. hirsutum and G. barbadense, and 14, 13, 27 and 23 PP2CA genes were identified in the genomic sequences of these plants, respectively. Analysis results showed that most Gossypium PP2CAs were highly conserved in chromosomal locations, structures and phylogeny among the four cotton species. Segmental duplication might play important roles in the formation of the PP2CAs, and most PP2CAs may be under purifying selection in Gossypium during evolution. The majority of the PP2CAs were expressed specifically in diverse tissues, and highly expressed in flowers in G. hirsutum. The GhPP2CAs displayed diverse expression patterns in responding to ABA and osmotic stress. Yeast-two hybrid assays revealed that many GhPP2CAs were capable of interaction with the cotton ABA receptor s pyrabactin resistance1/PYR1-like/regulatory components of ABA receptors (PYR1/PYL/RCAR) GhPYL2-2D (Gh_D08G2587), GhPYL6-2A (Gh_A06G1418) and GhPYL9-2A (Gh_A11G0870) in the presence and/or absence of $A B A$. These results gave a comprehensive view of the Gossypium PP2CAs, and are valuable for further studying the functions of PP2CAs in Gossypium. 
1 Title page

2 Title:

3 Genome-wide characterization and expression analysis of PP2CA family members in response to $\mathrm{ABA}$ and osmotic stress in Gossypium

6 Authors:

7 Tingting Lu ${ }^{1,2 \dagger}$, Gaofeng Zhang ${ }^{1 \dagger}$, Yibin Wang ${ }^{1}$, Shibin $\mathrm{He}^{1}$, Lirong Sun ${ }^{1^{*}}$ and Fushun

$8 \mathbf{H a o}^{\mathbf{1}^{*}}$

9 'State Key Laboratory of Cotton Biology, Henan Key Laboratory of Plant Stress Biology,

10 School of Life Sciences, Henan University, Kaifeng 475004, China

$11{ }^{2}$ Henan University of Animal Husbandry and Economy, Zhengzhou 450046, China

13 These authors contributed equally to this work

14 Tingting Lu: 4147505@qq.com

15 Gaofeng Zhang: 2604035350@qq.com

16 Yibin Wang: 1532231014@qq.com

17 Shibin He: Hsb108@163.com

18 "Corresponding author

19 Lirong Sun: sunlr9208@henu.edu.cn

20 Fushun Hao: haofsh@henu.edu.cn

$21 \quad$ Tel. 86-371-23881387; Fax 86-371-23881387 
22 Genome-wide characterization and expression analysis of PP2CA family members in response to

ABA and osmotic stress in Gossypium

Tingting $\mathrm{Lu}^{1,2 \dagger}$, Gaofeng Zhang ${ }^{1 \dagger}$, Yibin Wang ${ }^{1}$, Shibin $\mathrm{He}^{1}$, Lirong Sun ${ }^{1 *}$ and Fushun Hao ${ }^{1 *}$

${ }^{1}$ State Key Laboratory of Cotton Biology, Henan Key Laboratory of Plant Stress Biology, College of Life Sciences, Henan University, Kaifeng 475004, China

${ }^{2}$ Henan University of Animal Husbandry and Economy, Zhengzhou 450046, China

\section{ABSTRACT}

Clade A type $2 \mathrm{C}$ protein phosphatases (PP2CAs), as central regulators of abscisic acid (ABA) signaling, negatively control growth, development and responses to multiple stresses in plants. PP2CA gene families have been characterized at genome-wide levels in several diploid plants like Arabidopsis and rice. However, the information about genome organization, phylogenesis and putative functions of PP2CAs in Gossypium is lacking. Here, PP2CA family members were comprehensively analyzed in four Gossypium species including the diploid progenitor species $G$. arboretum, G. raimondii and the tetraploid species G. hirsutum and G. barbadense, and 14, 13, 27 and 23 PP2CA genes were identified in the genomic sequences of these plants, respectively. Analysis results showed that most Gossypium PP2CAs were highly conserved in chromosomal locations, structures and phylogeny among the four cotton species. Segmental duplication might play important roles in the formation of the $P P 2 C A s$, and most $P P 2 C A s$ may be under purifying selection in Gossypium during evolution. The majority of the PP2CAs were expressed specifically 
43 in diverse tissues, and highly expressed in flowers in G. hirsutum. The GhPP2CAs displayed

44 diverse expression patterns in responding to ABA and osmotic stress. Yeast-two hybrid assays

45 revealed that many GhPP2CAs were capable of interaction with the cotton suggested ABA

46 receptors pyrabactin resistance1/PYR1-like/regulatory components of ABA receptors

47 (PYR1/PYL/RCAR) GhPYL2-2D (Gh_D08G2587), GhPYL6-2A (Gh_A06G1418) and

48 GhPYL9-2A (Gh_A11G0870) in the presence and/or absence of ABA. These results gave a comprehensive view of the Gossypium PP2CAs, and are valuable for further studying the functions of these PP2CAs.

Keywords Gossypium; Clade A type 2C protein phosphatases (PP2CAs); gene family;

52 phylogeny; protein interaction

\section{INTRODUCTION}

Protein phosphorylation and dephosphorylation, as two central mechanisms of cellular signal transduction, play pivotal roles in many biological processes including growth, development and adaptations to various environmental stimuli in plants (Schweighofer et al., 2004). They are catalyzed by protein kinases and phosphatases, respectively. Phosphatases are generally categorized into serine/threonine (Ser/Thr) phosphatases and tyrosine (Tyr) phosphatases according to the different amino acid residues they dephosphorylate. Based on biochemical and

61 pharmacological properties, Ser/Thr phosphatases can be further classified into three large

62 families: phosphoprotein phosphatases (PPs), phosphoprotein metallophosphatases and aspartate-

63 based protein phosphatases (Schweighofer et al., 2004; Kerk et al., 2007; Fuchs et al., 2013; 
64 Singh et al., 2015). The PPs includes PP1, PP2A, PP2B, PP4, PP5, PP6 and PP7, the

65

66

67

68

69

phosphoprotein metallophosphatases consist of $\mathrm{Mg}^{2+} / \mathrm{Mn}^{2+}$-dependent type $2 \mathrm{C}$ protein

phosphatases (PP2Cs) and other $\mathrm{Mg}^{2+}$-dependent phosphatases (Schweighofer et al., 2004; Singh

et al., 2010; Fuchs et al., 2013; Singh et al., 2015). PP2Cs, which play key roles in

dephosphorylation events in plants, belong to a large subfamily, and can be further divided into

11 clades (A-K) in Arabidopsis and rice (Singh et al., 2010) and 12 clades (A-L) in

Brachypodium distachyon (Cao et al., 2016). Among these, clade A proteins of PP2Cs (PP2CAs)

are the ones of well-studied PP2Cs in Arabidopsis, and they have been shown to have important

roles in controlling abscisic acid (ABA) signaling, and negatively regulate plant growth,

development and response to various biotic and abiotic stresses in plants (Tähtiharju et al., 2001;

Fuchs et al., 2013; Singh et al., 2015). In Arabidopsis genome, 9 PP2CA members have been

identified. They are ABI1 (ABA insensitive 1), ABI2, HAB1 (Homology to ABI1), HAB2, AHG1

(ABA hypersensitive germination 1), HAII (Highly ABA-induced PP2C1), HAI2, HAI3 and

AHG3/AtPP2CA (Fuchs et al., 2013). These genes, particularly ABI1, ABI2 and AHG3/PP2CA

alone or cooperatively control ABA-mediated transpiration, stomatal closure, seed germination

and root growth, and are involved in the regulation of many abiotic stress responses like drought,

high salinity, cold, heat and potassium deprivation (Schweighofer et al., 2004; Rubio et al., 2009;

Singh et al., 2015). Some PP2CAs also play important roles in responses to pathogen attack

(Schweighofer et al., 2004; Singh et al., 2015). PP2CAs are functionally redundant, and their

expression is upregulated by high concentrations of ABA (Rubio et al., 2009; Singh et al., 2015).

Moreover, PP2CAs physically interact with numerous cytosolic and nuclear localized proteins 
such as AtHB6 (Homeobox protein 6), CIPK8 (Calcineurin B-like protein-interacting protein kinase 8), CIPK24, and SnRK2s (Sucrose nonfermenting 1-related protein kinases subfamily 2 proteins) (Ohta et al., 2003; Fuchs et al., 2013; Singh et al., 2015). SnRK2s exert central and positive roles in ABA signal cascade in plants (Fujii et al., 2009a; Fujita et al., 2009). Recently, ABA receptors pyrabactin resistance1/PYR1-like/regulatory components of ABA receptors (PYR1/PYL/RCAR) (named PYLs for simplicity) have been found (Ma et al., 2009; Park et al., 2009). This is a breathtaking discovery in plants. PP2CAs were identified as coreceptors, specifically interact with PYLs and control ABA signaling. In the presence of ABA, ABA binds to PYLs, further interacts with and inhibits the activities of PP2CAs; thereby releasing and activating SnRK2s. SnRK2s subsequently regulate multiple downstream transcriptional factors and other proteins to trigger ABA responses (Fujii et al., 2009b; Geiger et al., 2009; Lee et al., 2009).

Currently, PP2C gene families including PP2CAs have been analyzed at genome-wide levels in Arabidopsis, rice, maize and Brachypodium distachyon (Xue et al., 2008; Wei and Pan, 2014; Cao et al., 2016). The domain structure of PP2CAs was also studied (Schweighofer et al., 2004). Moreover, the expression patterns of $P P 2 C A s$ have been examined in response to ABA and multiple stresses in Arabidopsis, rice, maize and Brachypodium distachyon (Xue et al., 2008;

Wei and Pan, 2014; Zhang et al., 2017a). However, knowledge about the genomic information and expression profiles of PP2CAs in cotton is unknown to date.

Cotton is the most important fiber crop, which provides the spinnable lint for the textile industry in the world. The yield and quality of cotton are adversely affected by many abiotic 
106

107

108

109

110

111

112

113

114

115

116

117

118

119

120

121

122

123

124

125

126

17

stresses such as drought and high salinity, which are governed by ABA signaling (Hauser et al., 2011; Liang et al., 2017; Ullah et al., 2017). Therefore, it is essential for us to uncover the functional mechanisms of PP2CAs in ABA signal transduction pathway in cotton. Here, we carried out a genome-wide identification of PP2CA gene family in diploid G. arboretum (A2) and $G$. raimondii (D5), and their decendant tetraploid species $G$. hirsutum (AD1) and $G$. barbadense (AD2). The evolutionary relationships of these PP2CAs were analyzed. Changes in the transcriptional levels of the PP2CAs were also investigated in diverse tissues and in response to ABA and osmotic stress in G. hirsutum. Furthermore, the interactions between G. hirsutum PP2CAs and several GhPYLs were detected by the yeast-two hybrid method. These results may be valuable for further functional characterization of cotton PP2CAs in ABA signaling in the future.

MATERIALS AND METHODS

9 Analysis of the PP2C family in four Gossypium species

20 To explore all the members of the PP2C family in Gossypium, the protein sequences of 80 1 AtPP2Cs were initially applied as queries to search against the databases of G. arboreum (BGICGB v2.0 assembly genome), G. raimondii (JGI assembly v2.0 data.), G. hirsutum (NAU-NBI v1.1 assembly genome) (www.cottongen.org) and $G$. barbadense 24 (http://database.chgc.sh.cn/cotton/index.html), respectively, using the BLAST program with 25 default setting (E-value $\left.<\mathrm{e}^{-10}\right)($ Camacho et al., 2009). After removing the redundant sequences from the data set, the putative Gossypium PP2Cs were then characterized using the PP2C model 
127 (PF00481) (http://pfam.xfam.org/) by the Hmmer software (http://hmmer.org/), and the proteins

128 without a PP2C catalytic domain were deleted. The molecular weight (MW) and isoelectric point

129 (pI) of the Gossypium PP2Cs were predicted by the online tool ExPaSy

130 (http://web.expasy.org/protparam/), which can give various physico-chemical properties of a

131 protein based on its amino acid sequence (the extinction coefficient and the absorbance of a native

132 protein in water at $280 \mathrm{~nm}$ were used). The composition and position of exons and introns of the

133 PP2CAs were obtained from the website (https://www.cottongen.org/) and characterized by the

134 Gene Structure Display Server (GSDS) tools (http://gsds.cbi.pku.edu.cn/) (Hu et al., 2015). The

135 conserved domains of PP2CAs were validated in NCBI

136 (https://www.ncbi.nlm.nih.gov/Structure/cdd/wrpsb.cgi) using the automatic mode (Marchler-

137 Bauer et al., 2017). The MEME program (meme-suite.org/tools/meme) was applied to determine

138 the motifs of PP2CAs in Gossypium ('any number of repetitions' to be distributed in sequences

139 was set). The locations of Gossypium PP2CAs in chromosomes were assessed using the

140 MapInspect software (http:/www.mybiosoftware.com/mapinspect-compare-display-linkage-

141 maps.html).

142 Analysis of synteny and $K a / K s$ of PP2CAs

143 The homologous regions of PP2CAs in Gossypium were identified by the MCScanx software

144 (http://chibba.pgml.uga.edu/mcscan2/), and syntenic blocks were determined by the CIRCOS

145 program (http://www.circos.ca/). The syntenic maps of the $P P 2 C A s$ were obtained using the

146 circos- $0.69 \pm 3$ software with default parameters (http://www.circos.ca/). Some genes located 
147 within the same or adjacent intergenic region were regarded as tandem duplications. The

148 nucleotide substitution parameters $K a$ (non-synonymous) and $K s$ (synonymous) were assessed by

149 the PAML program (http://abacus.gene.ucl.ac.uk/software/paml.html). Then, the ratio of $K a / K s$

150 was calculated. $K a / K s<1$ means purifying selection; $K a / K s=1$ indicates neutral selection, while

$151 K a / K s>1$ represents positive selection (Hurst, 2002).

152

153

154

155

156

157

158

159

160

161

162

163

164

165

\section{Phylogenetic analysis of PP2CAs}

The PP2CA databases were downloaded for Arabidopsis thaliana (http://www.arabidopsis.org/),

Theobroma cacao (http://cocoagendb.cirad.fr), Ricinus communis (http://castorbean.jcvi.org),

Populus trichocarpa (http://www.phytozome.net/poplar), Glycine max

(http://www.phytozome.net/soybean), Brachypodium distachyon

(http://plants.ensembl.org/Brachypodium_distachyon/Info/Index ), Oryza sativa

(http://rapdb.dna.affrc.go.jp), and the four Gossypium species mentioned above. The amino acid sequences of PP2CAs were aligned by the MUSCLE software (Edgar et al., 2004), and a

phylogenetic tree of the PP2CAs was generated using the IQ-TREE server

(http://www.iqtree.org/) following the maximum likelihood (ML) method (Nguyen et al., 2015;

Trifinopoulos et al, 2016). The best-fitting model was chosen using the ModelFinder

(Kalyaanamoorthy et al. 2017) and the support was assessed using the ultrafast bootstrap (Minh et al., 2013). Evolutionary tree was visualized using the FigTree v1.4.4 software (available from https://github.com/rambaut/figtree/releases). 
166

167

168

169

\section{Measurements of GhPP2CAs expression in tissues and in response to ABA or osmotic stress}

For measuring the expression of GhPP2CAs in tissues in each experiment, seeds of G. hirsutum
L. acc. Texas Marker-1 (TM-1) were sown in pots containing the mixed nutrient soil (rich

soil:vermiculite $=2: 1, \mathrm{v} / \mathrm{v}$ ) in a growth chamber. After $21 \mathrm{~d}$, about $2 \mathrm{~g}$ samples of roots, stems or leaves were collected from 10 plants. About 20 flowers were collected $1 \mathrm{~d}$ post anthesis, and about $5 \mathrm{~g}$ fibers were obtained from ovules $23 \mathrm{~d}$ post anthesis of cotton plants grown in the

fields. For monitoring the expression of GhPP2CAs after ABA treatment or under osmotic stress,

TM-1 seeds were germinated and planted in liquid 1/2 MS medium (Murashige and Skoog, 1962) in a growth chamber (the medium was aerated. day/night temperature cycle of $28^{\circ} \mathrm{C} / 26^{\circ} \mathrm{C}$, $14 \mathrm{~h}$ light/10 h dark, and about 50\% relative humidity). Three weeks later, the plants were sprayed with $100 \mu \mathrm{M}$ ABA or treated with 10\% PEG6000 (dissolved in medium) for 0, 3, 6, 12 and $24 \mathrm{~h}$, respectively. Then, about $2 \mathrm{~g}$ roots were sampled, frozen in liquid nitrogen and stored at $-70^{\circ} \mathrm{C}$. Total RNA was extracted from some of the samples and cDNA was generated according to the method described previously (Ma et al., 2012; Zhang et al., 2017b).

Quantitative real-time RT-PCR (qRT-PCR) experiments were constructed in an ABI 7500 real-time PCR amplifier using the cDNA, SYBR Green Master mix, the specific primers of GhPP2CA genes (Table S1). GhUBQ7 was used as an internal control (Lu et al., 2017).

Experiments were independently repeated three times. The interval between two repeated experiments was 7-10 d.

\section{Monitoring protein interaction by yeast two-hybrid method}

The CDS sequences of GhPYLs (GhPYL2-2D, GhPYL6-2A, and GhPYL9-2A) and GhPP2CAs 
187 188

were amplified, and cloned into pGADT7 and pGBKT7 vectors respectively using gene specific primers (Table S2). After sequencing, the fused vectors were transformed into AH109. The cotransformants were plated on non-selective SD/-Leu/-Trp solid medium and selective SD/Leu/-Trp/-His/-Ade solid medium as described previously (Lu et al., 2017; Zhang et al., 2017b).

\section{RESULTS}

\section{Genome-wide analysis of PP2CAs in four Gossypium species}

To identify the putative PP2CA family members in Gossypium, the amino acid sequences of 80 Arabidopsis PP2Cs (Xue et al., 2008) were used to survey the Gossypium dababases. Putative PP2Cs were assigned to a total of $114,116,239$ and 232 genomic sequences that were retrieved from G. arboretum, G. raimondii, G. hirsutum and G. barbadense, respectively. They were individually denominated as GaPP2Cs, GrPP2Cs, GhPP2Cs and GbPP2Cs (Table S3). According to the phylogenetic relationships of PP2Cs between Gossypium and Arabidopsis, the Gossypium PP2Cs could be clustered into 12 clades (A-L) (Fig. S1-S4). The PP2CAs possessed 14 GaPP2CAs, 13 GrPP2CAs, 27 GhPP2CAs and 23 GbPP2CAs, respectively. They were named individually according to their gene identifiers (Table 1). In this report, we focused on the PP2CA family members in the four Gossypium species.

It was found that the predicted coded amino acid lengths of Gossypium PP2CAs ranged from 118 to 593 , with an average of 420 . These PP2CAs had molecular weights of $12.8 \mathrm{kDa}$ (GhPP2CA27) to $66 \mathrm{kDa}$ (GaPP2CA6). The mean theoretical pIs of PP2CAs was approximately 5.9 with a minimum of 4.65 (GrPP2CA5) and a maximum of 8.74 (GhPP2CA22) (Table 1). 


\section{Phylogenetic and structural analysis of PP2CAs in Gossypium}

209 In order to understand the evolutionary relationship among GaPP2CAs, GrPP2CAs, GhPP2CAs and GbPP2CAs, we conducted a phylogenetic tree using the protein sequences of the Gossypium PP2CAs (Fig. 1A). As expected, most of GaPP2CAs were individually clustered closely with their corresponding orthologs of GhPP2CAs (GhPP2CA1-13) and GbPP2CAs (GbPP2CA1-10) in A genomes, and a majority of GrPP2CAs individually clustered closely with their homologs of GhPP2CAs (GhPP2CA14-27) and GbPP2CAs (GbPP2CA11-23) in D genomes. Noteworthily, GaPP2CA10 clustered together with GaPP2CA11, and a similar case occurred between GbPP2CA21/GbPP2CA22 and GbPP2CA16/GbPP2CA23. Moreover, homologues of 14 GaPP2CAs and of 13 GrPP2CAs were found in the G. hirsutum At and Dt subgenomes, respectively; and homologs of $10 \mathrm{GaPP} 2 \mathrm{CAs}$ (except GaPP2CA1, GaPP2CA3, GaPP2CA4 and GaPP2CA14) and 11 GrPP2CAs (except GrPP2CA6 and GrPP2CA9) were detected in the $G$. barbadense At' or Dt' subgenomes, respectively. Additionally, three pairs of paralogues with high sequence similarity in GbPP2CAs including GbPP2CA8/GbPP2CA10, GbPP2CA16/GbPP2CA23, GbPP2CA21/GbPP2CA22 were clustered together. They were seemingly derived from GaPP2CA8, GrPP2CA7 and GrPP2CA1, respectively.

Most PP2CAs had 3-4 exons except that GaPP2CA6, GrPP2CA9, GhPP2CA22, GbPP2CA13

possessed 5 exons, and GhPP2CA27 had 2 exons. Among the PP2CA genes, GaPP2CA6, GrPP2CA9, GhPP2CA9 and GhPP2CA22 individually had a longer intron sequence than other genes did (Fig. 1B). These results indicate that the exon/intron structures of the Gossypium PP2CA genes were highly conserved. 
229 The motif compositions of the PP2CA proteins were analyzed in the four Gossypium species.

230 Twenty putative motifs named motif 1 to motif 20 were identified. Among those, motif 1, 2, 3, 4,

2315,6 and 7 existed in every cluster and the majority of the PP2CA members. Moreover, most

232 orthologous PP2CA proteins in the four Gossypium plants had the same or very similar

233 compositions and distributions of motifs, suggesting that the PP2CA members in the same

234 cluster likely share similar functions (Fig. 1C).

235 Chromosomal distributions of PP2CAs in Gossypium

236 To determine the putative evolutionary relationships of the Gossypium PP2CA genes, the positions

of the genes on chromosomes were analyzed. We found that the distributions of these PP2CAs

were uneven. The 14 GaPP2CAs, 13 GrPP2CAs, 27 GhPP2CAs and 23 GbPP2CAs were

distributed on 8, 9, 16 and 14 chromosomes, respectively. Most of the chromosomes contained

one PP2CA gene. By contrast, some chromosomes individually had two PP2CA genes. They were

D11 and D13 in G. raimondii, At10, At13, Dt05, Dt10 and Dt13 in G. hirsutum, and Dt'07 and

Dt'13 in G. barbadense. Besides, each of D09 and Dt'05 owned 3 PP2CAs. A13, At05 and At'05

separately possessed 4 PP2CAs. In contrast, GaPP2CA5, GaPP2CA8, GaPP2CA14, GhPP2CA18,

GhPP2CA20, GhPP2CA27, GbPP2CA2 and GbPP2CA12 were located on scaffolds, in which

contigs were not spliced into any chromosome in genomic mapping.

246 We compared the positions of the orthologs of GaPP2CAs, GrPP2CAs and GhPP2CAs or

GbPP2CAs in chromosomes. As expected, most homologs of GaPP2CAs and GrPP2CAs in G.

hirsutum were located in their corresponding At subgenomes and Dt subgenomes. The similar

249 situation also occurred in G. barbadense. However, homologous genes of GaPP2CAs and 
$250 \mathrm{GrPP} 2 \mathrm{CAs}$ were barely located in their corresponding homoeologous chromosomes and collinear

251 loci in G. hirsutum and G. barbadense (Fig. 2). For example, GaPP2CA7 was found in

252 chromosome A09, however, its ortholog GhPP2CA3 and GbPP2CA9 were present in chromosome

253 At05 and At'05, respectively. These results imply that specific, unique and complex variation

254 events in PP2CA-contained homoeologous chromosomes may happen within each of the two

255 diploid and tetraploid species during genetic evolution.

256

257

258

259

260

261

262

263

264

265

266

267

268

269

270

\section{Synteny analysis of $P P 2 C A$ genes}

During evolutionary processes, tandem and segmental duplications contribute to expanding gene family in plants (Cannon et al., 2004). We examined the duplication relationship of the PP2CAs among G. arboretum, G. raimondii and G. hirsutum (the related database for G. barbadense was lacking). It was found that GaPP2CA10 and GaPP2CA11 joined together, and GbPP2CA16 and GbPP2CA23 clustered together in the chromosome. There are less than 5 genes between each pair of the genes, suggesting that the two pairs of genes are tandemly duplicated.

The synteny relationship of gene pairs was also explored among GaPP2CAs, GrPP2CAs and GhPP2CAs. A total of 136 homologous gene pairs were observed in 133 collinearity blocks. Most of the blocks had one gene pair. Some blocks owned two gene pairs (GrPP2CA9/GhPP2CA22, GrPP2CA10/GhPP2CA23) between chromosomal D11 and Dt10. Another block harbored three gene pairs (GrPP2CA5/GhPP2CA2, GrPP2CA6/GhPP2CA3, GrPP2CA7/GhPP2CA4) between chromosomal D09 and At05 (Fig. 3). These findings imply that segmental duplication plays major roles in generating PP2CAs during evolution in Gossypium.

\section{Analysis of $K a / K s$ values of $P P 2 C A s$}


271 To further understand the evolution processes among Gossypium PP2CAs, the effects of selection

272 on duplication of $P P 2 C A$ genes were determined. The non-synonymous $(K a)$ and synonymous

$273(K s)$ substitutions, and $K a / K s$ values were calculated for the homologous gene pairs among

$274 G a P P 2 C A s, G r P P 2 C A s$ and GhPP2CAs. The mean values of $K a / K s$ for these gene pairs between

275 species Ga/Gh, Gr/Ga, Gr/Gh, Ga/Ga, Gr/Gr, Gh/Gh were 0.22, 0.21, 0.22, 0.21, 0.19 and 0.21,

276 respectively. All of them were less than 1, indicating that the formation of these genes were mainly

277 under purifying selection during evolution. The $K a / K s$ ratios for the two gene pairs

278 GrPP2CA11/GhPP2CA15 and GrPP2CA3/GhPP2CA21 were higher than 1, suggesting that the

279 two gene pairs were generated under positive selection and the selection likely has effects to

280 change these genes during evolution (Fig. 4).

281 Phylogenetic analysis of PP2CAs in Gossypium and other plants

282 We constructed a phylogenetic tree of PP2CA proteins in G. arboretum, G. raimondii, G. hirsutum,

G. barbadense, A. thaliana, T. cacao, R. communis, P. trichocarpa, G. max, B. distachyon and $O$.

sativa using the maximum likelihood (ML) method, and analyzed the evolutionary relationships

of these PP2CAs. It was found that the PP2CAs included both dicotyledonous and

monocotyledonous members (Fig. 5). This suggests that these PP2CAs formed before the

divergence of eudicots and monocots and are in general highly conserved. Indeed, the PP2CAs

from the eudicots Gossypium, cacao, poplar, castor, soybean and Arabidopsis clustered more

closely, and those of the monocots rice and distachyon clustered together. Moreover, many

PP2CAs from Gossypium clustered more closely with those from cacao than from poplar, castor, 
292

293

with those of cacao than those of other plants. As expected, PP2CAs in the four Gossypium species always clustered together, in line with their homologous evolutionary relationships (Fig. 5).

\section{Expression patterns of $G h P P 2 C A$ genes in different tissues}

The transcript abundances of 27 GhPP2CA in various tissues were measured by qRT-PCR to determine the putative functions of the PP2CAs in cotton. The results showed that all of the GhPP2CAs were highly expressed in flowers. GhPP2CA11 and GhPP2CA27 were also preferentially expressed in roots. Moreover, the transcriptional levels of GhPP2CA3, 11, 13, 27 were high in fibers. The transcripts of $G h P P 2 C A 4,16,22$ were abundant in stems. These results imply that most cotton PP2CA members may function in reproductive development, and some PP2CAs also play roles in some specific tissues like roots, fibers and stems (Fig. 6).

\section{Transcriptional changes of $G h P P 2 C A s$ in responses to $\mathrm{ABA}$ and osmotic stress}

To gain insight into the roles of GhPP2CAs in ABA signaling, transcriptional abundances of

GhPP2CAs in roots were detected after treatments with $100 \mu \mathrm{M}$ ABA or $10 \%$ PEG6000 for indicated periods of time. We observed that the transcriptional levels of some GhPP2CA genes such as $G h P P 2 C A 5,11,18,20,25,27$ continually increased with the extension of ABA treatment time. In contrast, the expression levels of some members including GhPP2CA2-4, 8, 10, $24 \mathrm{had}$ decreasing trend. The expression levels of some genes were decreased at $3 \mathrm{~h}$ or $6 \mathrm{~h}$ but increased at $12 \mathrm{~h}$ or $24 \mathrm{~h}$. These genes included GhPP2CA1, 16, 17, 19, 26. The expression levels of several genes increased at $3 \mathrm{~h}$ or $6 \mathrm{~h}$ but decreased at $12 \mathrm{~h}$ or $24 \mathrm{~h}$. These genes were GhPP2CA6, 7, 9, 12-15, 21-23 (Fig. 7). Treatment of cotton seedlings with PEG6000 also altered the expression of most GhPP2CA genes (Fig. 8). The majority of GhPP2CAs were 
313 upregulated after treatments with PEG for a short time period, and downregulated afterwards.

314 For example, the transcriptional levels of GhPP2CA8 and GhPP2CA21 were prominently

enhanced at $3 \mathrm{~h}$, and then reduced at $6 \mathrm{~h}, 12 \mathrm{~h}$ and $24 \mathrm{~h}$, while those of GhPP2CA5, 6, 17, 23, 25,

26 were pronouncedly increased at $6 \mathrm{~h}$ and decreased at $12 \mathrm{~h}$ and $24 \mathrm{~h}$. By contrast, the

expression of some genes was significantly elevated at 12 or $24 \mathrm{~h}$ post PEG treatment. These

genes included $G h P P 2 C A 1,3,4,7,9,12-14,18,20,24,27$. The overall trend of expression of

GhPP2CA10 and GhPP2CA15 was increased while that of GhPP2CA2, 11, 16 was decreased

under osmotic stress (Fig. 8). Together, these data suggest that GhPP2CAs exhibit diverse

expression patterns in responses to ABA and osmotic stress.

322

323

324

325

326

327

328

329

330

331

332

333

\section{Many GhPP2CAs interact with GhPYL2-2D, GhPYL6-2A and GhPYL9-2A}

PP2CAs have been documented to interact with ABA receptor PYLs in ABA signal pathway

(Ma et al., 2009; Park et al; 2009). Accordingly, we investigated the interactions between

GhPP2CAs and GhYPLs in the absence or presence of ABA by yeast-two hybrid method. A total of 11 GhPP2CAs were cloned, and three GhPYLs GhPYL2-2D (Gh_D08G2587), GhPYL6-2A

(Gh_A06G1418) and GhPYL9-2A (Gh_A11G0870) were randomly selected and cloned. These genes were fused into yeast vectors, and yeast-two hybrid experiments were performed. In the absence of ABA, GhPP2CA2 and GhPP2CA25 respectively interacted with GhPYL2-2D while multiple GhPP2CAs like GhPP2CA2, 3, 6, 10, 13, 15, 18, 19, 25 individually interplayed with GhPYL2-2D in the presence of ABA (Fig. 9). In contrast, several GhPP2CAs could respectively interact with GhPYL6-2A or GhPYL9-2A either with or without ABA. These GhPP2CAs included GhPP2CA2, 6, 10, 13, 15, 18, 19, 24, 25. Besides, GhPP2CA3 interact with GhPYL9- 
334 2A but not with GhPYL6-2A either in the presence or absence of ABA (Fig.10, Fig. 11). These

335 results imply that GhPP2CAs differentially interact with GhPYLs in responding to ABA in

336 cotton.

337

338 DISCUSSION

339 PP2CAs are central components of ABA signal transduction pathway, and negatively control

340 ABA and stress responses in plants (Fuchs et al., 2013; Singh et al., 2015). They have been

341 identified in several plants including Arabidopsis, rice, maize and Brachypodium distachyon in

342 recent years (Xue et al., 2008; Wei and Pan, 2014; Cao et al., 2016). However, phylogenesis and

343 putative functions of PP2CAs in Gossypium remain elusive. In the present study, 14, 13, 27 and

34423 PP2CA genes were characterized in genomes of G. arboretum, G. raimondii, G. hirsutum and

345 G. barbadense, respectively (Table 1). Compared to the number of PP2CAs in Arabidopsis (9),

346 rice (10), maize (16) and Brachypodium distachyon (8), that in G. hirsutum and G. barbadense

347 was great (Xue et al., 2008; Wei and Pan, 2014; Cao et al., 2016). This suggests that more

348 complex and elaborate ABA signaling mechanisms modulated by PP2CAs may exist in the

349 upland and island cotton species. Conceivably, the high number of PP2CAs of the two species is

350 related to their tetrapoid nature. The two plants retain most PP2CA homologs of both $G$.

351 arboreum and G. raimondii but not a copy of either one progenitor during evolution. This may

352 be due to long-term human selection with these two tetraploid cotton species for higher yields,

353 growth in hotter and drier regions, day neutral flowering, and adaptation to agronomic areas far

354 outside their original habitats. These altered characteristics may be associated with more PP2CA 
355

356

357

358

359

360

361

362

363

364

365

366

367

368

369

370

371

372

373

374

375

proteins and complex ABA signal mechanisms in the cultivated cotton plants than in wild plants

and in the greater opportunities to accumulate sequences, amenable to mutation and selection, in a tetraploid genome than in a diploid genome.

We noticed that 27 GhPP2CAs and 23 GbPP2CAs individually had their corresponding orthologs in G. arboretum or G. raimondii (Fig. 1), indicating that those PP2CAs in G. hirsutum and $G$. barbadense are ancestrally related to those PP2CAs in the two diploid species.

Additionally, no orthologous genes of GaPP2CA1, 3, 4, 14, GrPP2CA6 and GrPP2CA9 were observed in G. barbadense (Fig. 1). This hints that these genes are possibly lost, or these genes arose after the tetraploid species appeared and separated from the diploid species during the evolutionary processes.

The structures and the numbers of introns and exons in PP2CAs were similar among the 4 Gossypium species as well as Arabidopsis, rice, maize and Brachypodium distachyon (Xue et al., 2008; Wei and Pan, 2014; Cao et al., 2016), suggesting that the PP2CAs undergo conserved evolutionary processes even after the divergence of monocotyledons and dicotyledons.

Colinearity results showed that 136 homologous gene pairs existed among GaPP2CAs, GrPP2CAs and GhPP2CAs (Fig. 3), indicating that PP2CA genes expand primarily through segmental duplication of DNA. Segmental duplicates may be more often maintained through subsequent gene subfunctionalization compared to tandem duplicates (Lynch and Conery, 2000). Accordingly, these PP2CAs probably had diverse functions in Gossypium. Moreover, in agreement with our results, Arabidopsis phosphatase family genes also showed segmental duplication (Cannon et al., 2004), suggesting the evolutionary mechanism of PP2CAs may be 
376

377

378

379

380

381

382

383

conserved in plants. The mean value of $K a / K s$ for a majority of $P P 2 C A$ homologous gene pairs was about 0.2 , significantly less than 1 (Fig. 4). This hints that most mutations occurred in the genomic sequences of PP2CAs in G. arboretum, G. raimondii and G. hirsutum during evolution were detrimental for plant survival, or the mutations-caused traits were not required for man. Thus these mutated genes were gradually eliminated, and only those we found were kept during the long time selection.

Phylogenetic results showed that the PP2CA members from monocotyledonous plants clustered together, and similar results occurred in dicotyledonous PP2CAs (Fig. 5). This suggests that great changes in DNA sequences of the $P P 2 C A s$ have taken place after isolation of monocotyledons and dicotyledons although these genes shared a common ancestor. PP2CAs in Gossypium always clustered together with those in T. cacao rather than with those in A. thaliana, R. communis, P. trichocarpa, G. max, B. distachyon and O. sativa (Fig. 5), pointing to the closer evolutionary relationship of Gossypium with T. cacao. That is, most of homologous PP2CA members in Gossypium and cacao were generated before separation of the two genera from the common ancestor. The common PP2CAs across all plants show that they still have core functions essential to basic plant survival and functions. Differences in PP2CAs that follow differentiation of different genera and even species show that they are still diverse and can accommodate functions specific to the survival of species and even in response to selection by man. 'Housekeeping' PP2CAs could probably be subtracted from the picture to illuminate the more unique ones to better understand functions of individual PP2CAs and their roles in specific species, traits, or even agronomic performance of specific cultivars. 
Transcript abundance analysis indicated that the majority of the GhPP2CAs was

predominantly expressed in flowers (Fig. 6), suggesting that GhPP2CA-mediated ABA signaling may be of great importance in flower development of cotton. High expression of GhPP2CAs in flowers was likely due to the importance of timing flowering to environmental conditions of native Gossypium plants. Because evolution of some species is tied to long-term human selection of cotton plants with high yields of fibers and good adaptations to hot and dry growth conditions.

Cotton yields are closely associated with flowering in agronomic conditions created by man that often are much different from the natural habitats of wild or ancestral Gosssypium species (e.g. cultivation only in summers of termperate-tropical latitudes instead of perennial growth in tropical latitudes closer to the equator). Drought and hot stresses should limit flower development. PP2CAs are negative regulators of the adverse stresses; and therefore, may facilitate flowering of cotton in these newer enviroments. The expression of most GhPP $2 C A s$ was upregulated after treatment with ABA or PEG6000 (Fig. 7, Fig. 8), in good agreement with the results from $A t P P 2 C A s, O s P P 2 C A s$ and $B d P P 2 C A s$ (Xue et al. 2008; Cao et al. 2016). These findings imply that PP2CAs are essential for plant response to ABA and osmotic stress. that most GhPP2CAs can individually interact with the three GhPYLs in the absence or presence

414 of ABA (Fig. 9-11). GhPYLs are homologs of AtPYLs and some GhPYLs have been suggested

415 to be functional ABA receptors (Liang et al., 2017; Zhang et al., 2017b). These data indicate that 416 a large number of GhPP2CAs may play roles via interactions with GhPYLs in ABA-dependent 
418 signaling will be further explored in the future.

\section{CONCLUSIONS}

420 In total, 14, 13, 27 and 23 PP2CA genes were characterized from G. arboretum, G. raimondii, G.

421 hirsutum and G. barbadense, respectively. These genes shared high similarity in chromosomal

422 locations, structures and phylogeny among the species. Most of them might be under purifying

423 selection during evolution. Moreover, PP2CAs displayed specific expression patterns in tissues

424 and diverse expression profiles in response to ABA and osmotic stress in G. hirsutum. Yeast-two

425 hybrid experiments indicated that most GhPP2CAs interacted with GhPYL2-2D, GhPYL6-2A

426 and GhPYL9-2A with or without ABA. These findings provide essential information for in-

427 depth investigations of the functions of PP2CAs in Gossypium in the future.

428

429 ADDITIONAL INFORMATION AND DECLARATIONS

$430 \quad$ Funding

431 This work was supported by Foundation of Program for young backbone teachers in universities

432 of Henan Province [2016GGJS-024], the scientific research and innovation team in Henan

433 University of Animal Husbandry and Economy (2018KYTD18), and the "111" Project. The

434 funders had no role in study design, data collection and analysis, decision to publish, or

435 preparation of the manuscript.

\section{Grant Disclosures}

437 The following grant information was disclosed by the authors:

438 Program for young backbone teachers in universities of Henan Province: 2016GGJS-024. 
439 Foundation of scientific research and innovation team in Henan University of Animal Husbandry

440 and Economy: (2018KYTD18).

441 "111" Project.

\section{Competing Interests}

443 The authors declare there are no competing interests.

\section{Author Contributions}

445 - Tingting Lu and Gaofeng Zhang analyzed the data and performed the experiments,

446 - Yibin Wang and Shibin He contributed to prepare various reagents/materials/analysis tools.

447 - Lirong Sun and Fushun Hao conceived and designed the experiments, and wrote the

448 manuscript.

\section{REFERENCES}

451

452

Camacho C, Coulouris G, Avagyan V, Ma N, Papadopoulos J, Bealer K, Madden LT. 2009. Blast+: architecture and applications. BMC Bioinformatics 10: 421.

Cannon SB, Mitra A, Baumgarten A, Young ND, May G. 2004. The roles of segmental and tandem gene duplication in the evolution of large gene families in Arabidopsis thaliana. BMC Plant Biol 4:10.

Cao J, Jiang M, Li P, Chu Z. 2016. Genome-wide identification and evolutionary analyses of the PP2C gene family with their expression profiling in response to multiple stresses in Brachypodium distachyon. BMC Genomics 17:175.

Edgar RC. 2004. Muscle: multiple sequence alignment with high accuracy and high throughput. 
Nucleic Acids Res 32:1792-1797.

461

Fuchs S, Grill E, Meskiene I, Schweighofer A. 2013. Type 2C protein phosphatases in plants. FEBS Journal 280:681-693.

Fujii H, Zhu JK. 2009a. Arabidopsis mutant deficient in 3 abscisic acid-activated protein kinases reveals critical roles in growth, reproduction, and stress. Proceedings of the National Academy of Sciences 106:8380-8385.

Fujii H, Chinnusamy V, Rodrigues A, Rubio S, Antoni R, Park SY, Cutler SR, Sheen J, Rodriguez PL, Zhu JK. 2009b. In vitro reconstitution of an abscisic acid signalling pathway. Nature 462:660-664.

Fujita Y, Nakashima K, Yoshida T, Katagiri T, Kidokoro S, Kanamori N, Umezawa T, Fujita M, Maruyama K, Ishiyama K, Kobayashi M, Nakasone S, Yamada K, Ito T, Shinozaki K, Yamaguchi-Shinozake K. 2009. Three SnRK2 protein kinases are the main positive regulators of abscisic acid signaling in response to water stress in Arabidopsis. Plant and Cell Physiology 50:2123-2132.

Geiger D, Scherzer S, Mumm P, Stange A, Marten I, Bauer H, Ache P, Matschi S, Liese A, AI-Rasheid KA, Romeis T, Hedrich R. 2009. Activity of guard cell anion channel SLAC1 is controlled by drought-stress signaling kinase-phosphatase pair. Proceedings of the National Academy of Sciences 106:21425-21430.

Hauser F, Waadt R, Schroeder J. 2011. Evolution of Abscisic Acid Synthesis and Signaling Mechanisms. Current Biology 21:346-355.

Hu B, Jin J, Guo AY, Zhang H, Luo J, Gao G. 2015. GSDS 2.0: an upgraded gene feature 

visualization server. Bioinformatics 31: 1296-1297.

482

Hurst LD. 2002. The Ka/Ks ratio: diagnosing the form of sequence evolution. Cell 18:486.

Kalyaanamoorthy S, Minh BQ, Wong TKF, vib Haeseker A, Jermiin LS. 2017. ModelFinder: fast model selection for accurate phylogenetic estimates. Nature Methods 14:587-589.

Kerk D, Templeton G, Moorhead GBG. 2007. Evolutionary radiation pattern of novel protein phosphatases revealed by analysis of protein data from the completely sequenced genomes of humans, green algae, and higher plants. Plant Physiology 146:351-367.

Lee SC, Lan W, Buchanan BB, Luan S. 2009. A protein kinase-phosphatase pair interacts with an ion channel to regulate ABA signaling in plant guard cells. Proceedings of the National Academy of Sciences 106:21419-21424.

\section{Liang C, Liu Y, Li Y, Meng Z, Yan R, Zhu T, Wang Y, Kang S, Abid MA, Malik W, Sun} G, Guo S, Zhang R. 2017. Activation of ABA receptors gene GhPYL9-11A is positively correlated with cotton drought tolerance in transgenic Arabidopsis. Frontiers in Plant Science 8: 1453.

Lu TT, Zhang GF, Sun LR, Wang J, Hao FS. 2017. Genome-wide identification of CBL family and expression analysis of CBLs in response to potassium deficiency in cotton. PeerJ 5:e3653.

Lynch M, Conery JS. 2000. The evolutionary fate and consequences of duplicate genes. Science 290:1151-1155. 
502

503

504

505

506

507

508

Ma LY, Zhang H, Sun LR, Jiao YH, Zhang GZ, Miao C, Hao FS. 2012. NADPH oxidase AtrbohD and AtrbohF function in ROS-dependent regulation of $\mathrm{Na}^{+} / \mathrm{K}^{+}$homeostasis in Arabidopsis under salt stress. Journal of Experimental Botany 63:305-317.

Marchler-Bauer A, Bo Y, Han L, He J, Lanczycki CJ, Lu S, Chitsaz F, Derbyshire MK, Geer RC, Gonzales NR, Gwadz M, Hurwitz DI, Lu F, Marchler GH, Song JS, Thanki N, Wang Z, Yamashita RA, Zhang D, Zheng C, Geer LY, Bryant SH. 2017. CDD/SPARCLE: functional classification of proteins via subfamily domain architectures. Nucleic Acids Research 45: D200-D203.

Ma Y, Szostkiewicz I, Korte A, Moes A, Yang Y, Christmann A, Grill E. 2009. Regulators of PP2C phosphatase activity function as abscisic acid sensors. Science 324:1064-1068.

Minh BQ, Nguyen MA, von Haeseler A. 2013. Ultrafast approximation for phylogenetic bootstrap. Molecular\& Biology\& Evolution 30:1188-1195.

Murashige T, Skoog F. 1962. A revised medium for rapid growth and bio-assays with tobacco tissue culture. Physiologia Plantarum 15:473-497.

Nguyen LT, Schmidt HA, Von Haeseler A, Minh BQ. 2015. IQ-TREE: a fast and effective stochastic algorithm for estimating maximum-likelihood phylogenies. Molecular Biology and Evolution 32:268-274.

Ohta M, Guo Y, Halfter U, Zhu J. 2003. A novel domain in the protein kinase SOS2 mediates interaction with the protein phosphatase 2C ABI2. Proceedings of the National Academy of Science 100:11771-11776.Park SY, Fung P, Nishimura N, Jensen DR, Fujii H, Zhao Y, Lumba S, Santiago J, Rodrigues A, Chow TF, Alfred SE, Bonetta D, Finkelstein R, 


\section{Provart NJ,Desveaux D, Rodriguez PL, McCourt P, Zhu JK, Schroeder JI, Volkman}

BF, Cutler SR. 2009. Abscisic acid inhibits type $2 \mathrm{C}$ protein phosphatases via the PYR/PYL family of START proteins. Science 324:1068-1071.

\section{Rubio S, Rodrigues A, Saez A, Dizon MB, Galle A, Kim TH, Santiago J, Flexas J,}

Schroeder JI, Rodriguez PL. 2009. Triple loss of function of protein phosphatases type 2C leads to partial constitutive response to endogenous abscisic acid. Plant Physiol 150:13451355.

Schweighofer A, Hirt H, Meskiene I. 2004. Plant PP2C phosphatases: emerging functions in stress signaling. Trends in Plant Science 9:236-243.

Singh A, Giri J, Kapoor S, Tyagi AK, Pandey GK. 2010. Protein phosphatase complement in rice: genome-wide identification and transcriptional analysis under abiotic stress conditions and reproductive development. BMC Genomics 11:435.

Singh A, Pandey A, Srivastava AK, Tran LS, Pandey GK. 2015. Plant protein phosphatases $2 \mathrm{C}$ : from genomic diversity to functional multiplicity and importance in stress management. Critical Reviews in Biotechnology 36:1023-1035.

Tähtiharju S, Palva T. 2001. Antisense inhibition of protein phosphatase $2 \mathrm{C}$ accelerates cold acclimation in Arabidopsis thaliana. The Plant Journal 26: 461-470.

Trifinopoulos J, Nguyen LT, Von Haeseler A, Minh BQ. 2016. W-IQ-TREE: a fast online phylogenetic tool for maximum likelihood analysis. Nucleic Acids Research 44:232-235.

Ullah A, Sun H, Yang X, Zhang X. 2017. Drought coping strategies in cotton: increased crop per drop. Plant Biotechnology Journal 15: 271-284. 
544 Wei K, Pan S. 2014. Maize protein phosphatase gene family: identification and molecular 545 characterization. BMC Genomics 15:773.

546 Xue T, Wang D, Zhang S, Ehlting J, Ni F, Jakab S, Zheng C, Zhong Y. 2008. Genome-wide 9:550.

Zhang F, Wei Q, Shi J, Jin X, He Y, Zhang Y, Luo Q, Wang Y, Chang J, Yang G, He G. 2017a. Brachypodium distachyon BdPP2CA6 interacts with BdPYLs and BdSnRK2 and positively regulates salt tolerance in transgenic Arabidopsis. Frontiers in Plant Science 8:264.

Zhang GF, Lu TT, Miao WW, Sun LR, Tian M, Wang J, Hao FS. 2017b. Genome-wide identification of ABA receptor PYL family and expression analysis of PYLs in response to ABA and osmotic stress in Gossypium. PeerJ 5:e4126. 
Figure 1

Phylogenetic relationships, gene structures and conserved motifs of PP2CA genes in Gossypium .

( A ) The phylogenetic tree was generated by the maximum likelihood (ML) method, with ultrafast bootstrap. (B ) Exon/intron architectures of Gossypium PP2C genes. The black boxes indicate exons, and the black lines represent introns. The sizes of exons and introns were determined by the scale at the bottom. (C) Distributions of conserved motifs. The motifs are displayed by 20 different color boxes.
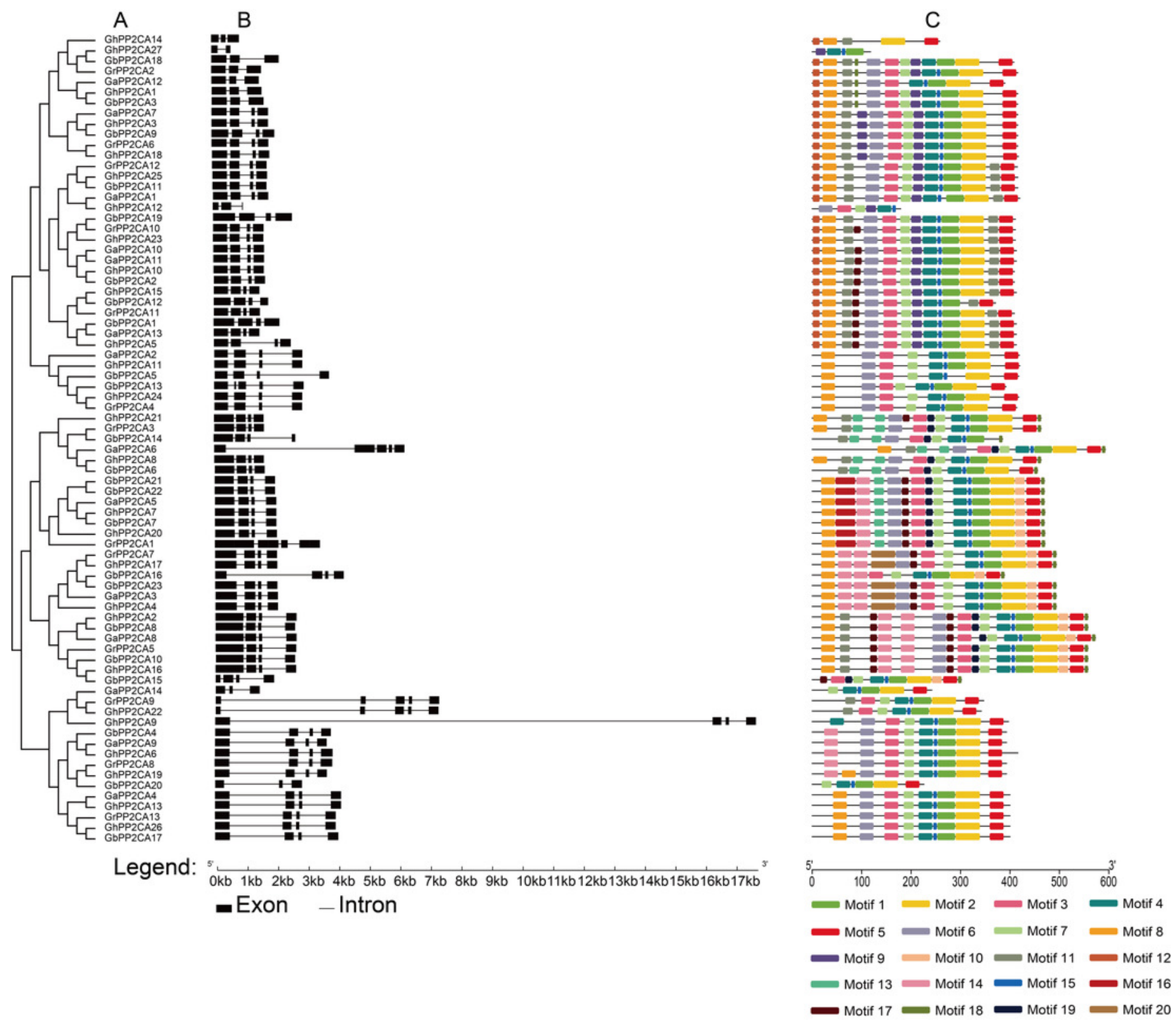
Figure 2

Positions of Gossypium PP2CA genes on chromosomes.

GaPP2CAs , GrPP2CA s GhPP2CA s, and GbPP2CAs were from G. arboreum, G. raimondii , G. hirsutum, and $G$. barbadense, respectively. 

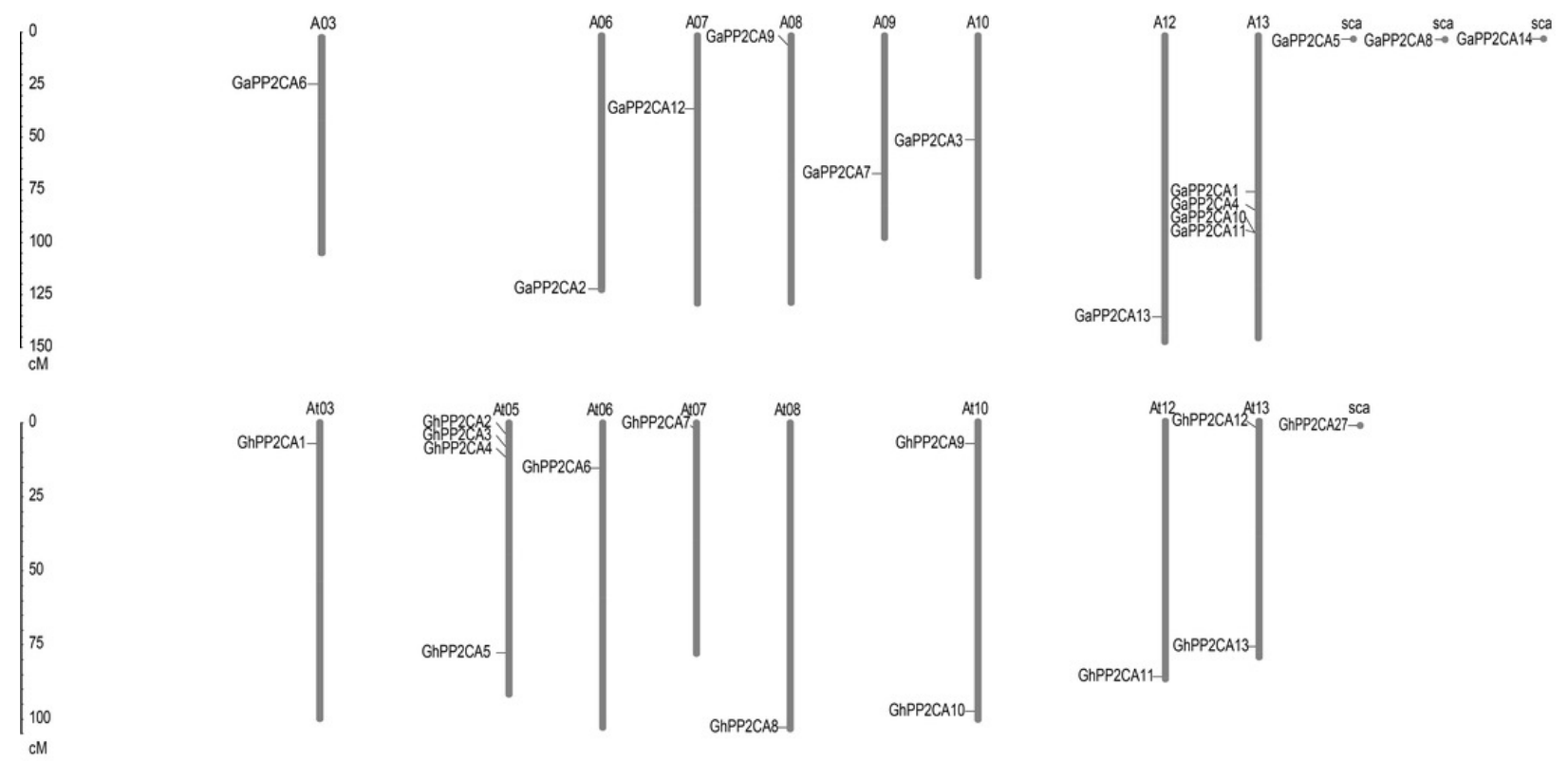

$\left.\right|^{0} 25$
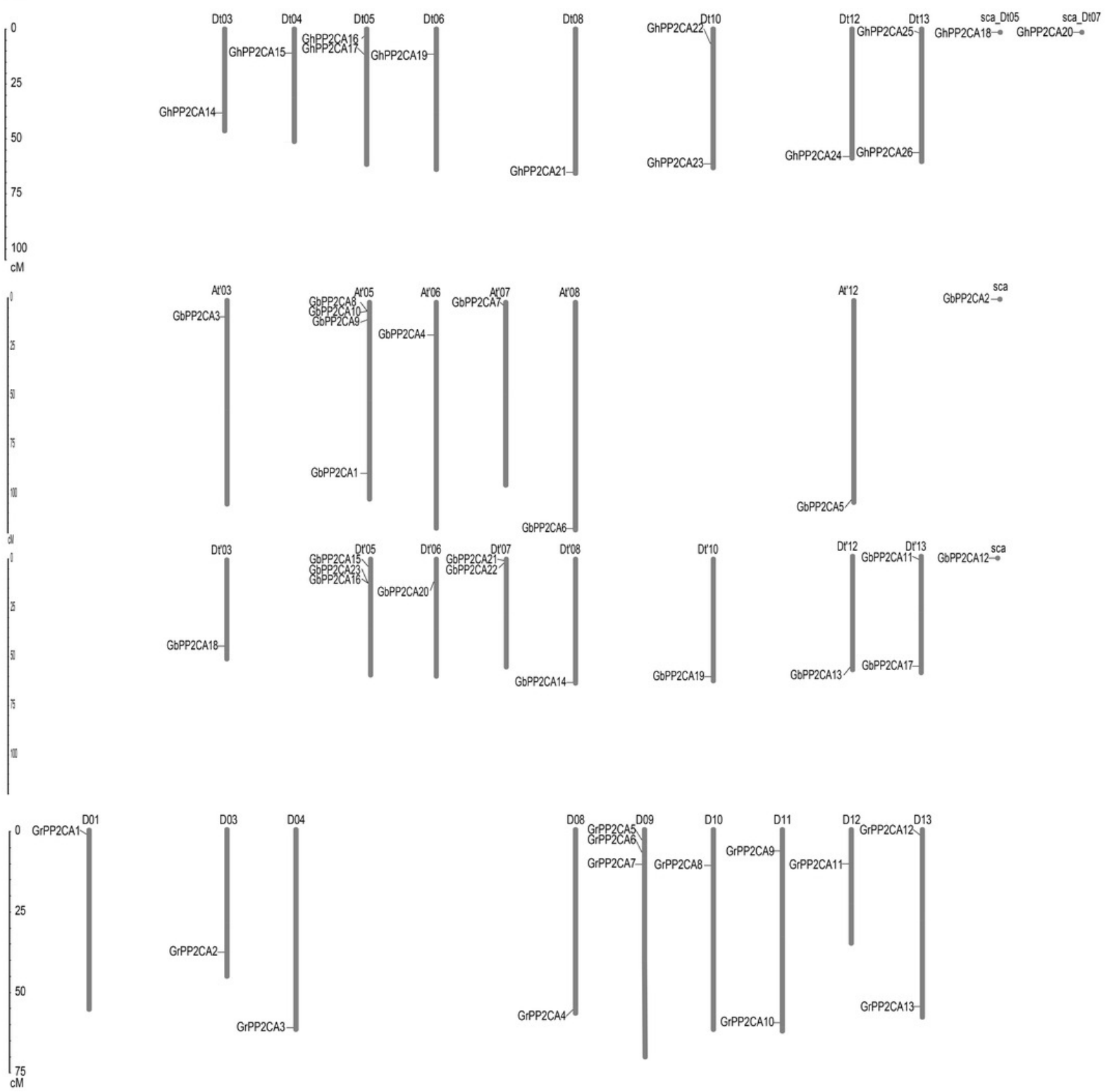

PeerJ reviewing PDF | (2018:11:32799:2:0:NEW 2 May 2019) 


\section{Figure 3}

Genome-wide synteny results of PP2CA genes from G.arboreum, G. raimondii and G. hirsutum.

Green lines linked gene pairs between $G$. arboreum and G. hirsutum, red lines connected gene pairs between $G$. raimondii and $G$. hirsutum, blue lines bridged gene pairs between $G$. arboreum and $G$. raimondii, yellow lines joined gene pairs within individual species in $G$. arboretum, $G$. raimondii and $G$. hirsutum. 


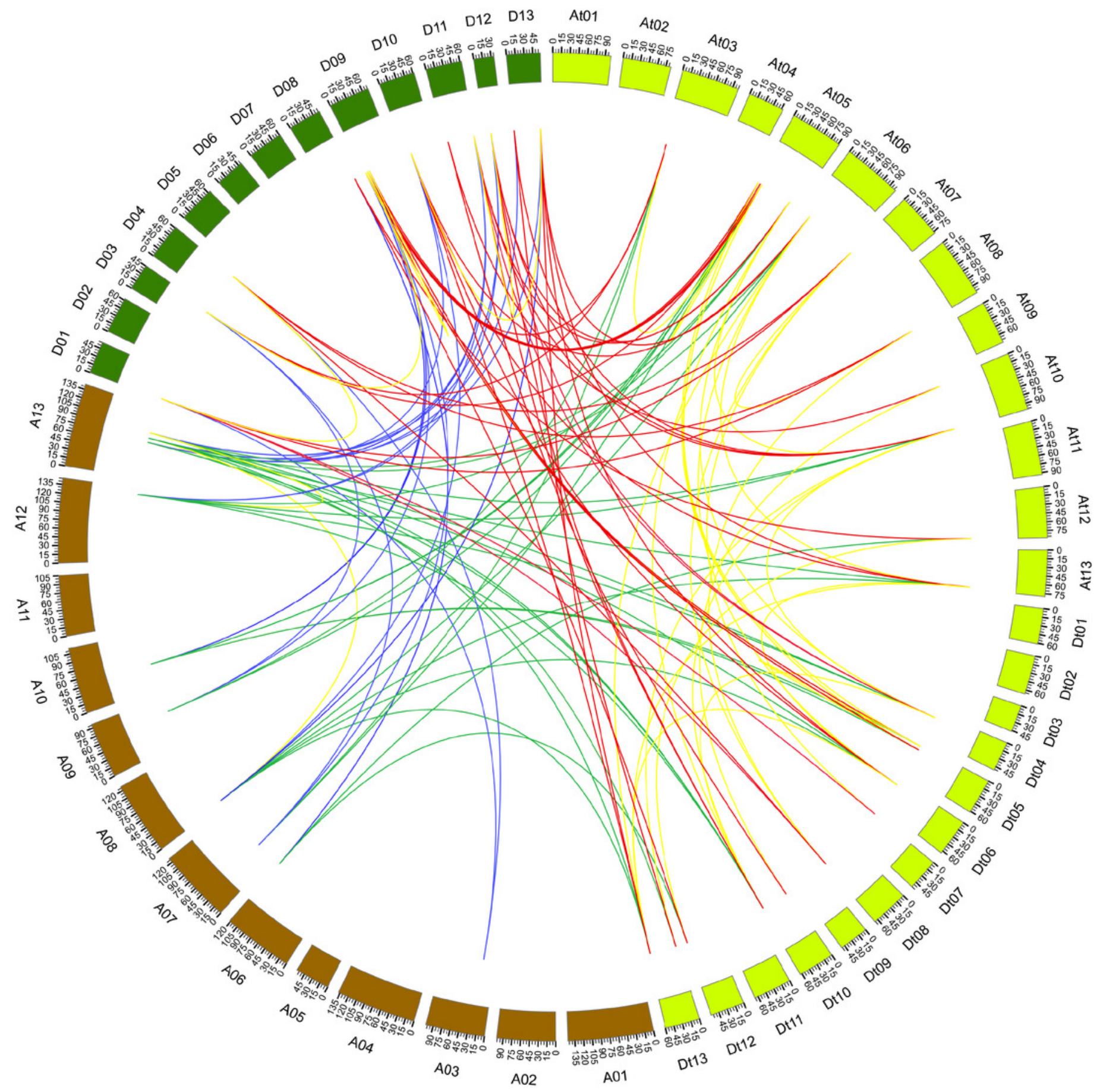


Figure 4

The Ka/Ks values of the homologous PP2CA gene pairs in Gossypium.

(Ga), (Gr) and (Gh ) represented G. arboretum, G. raimondii, and G. hirsutum, respectively.

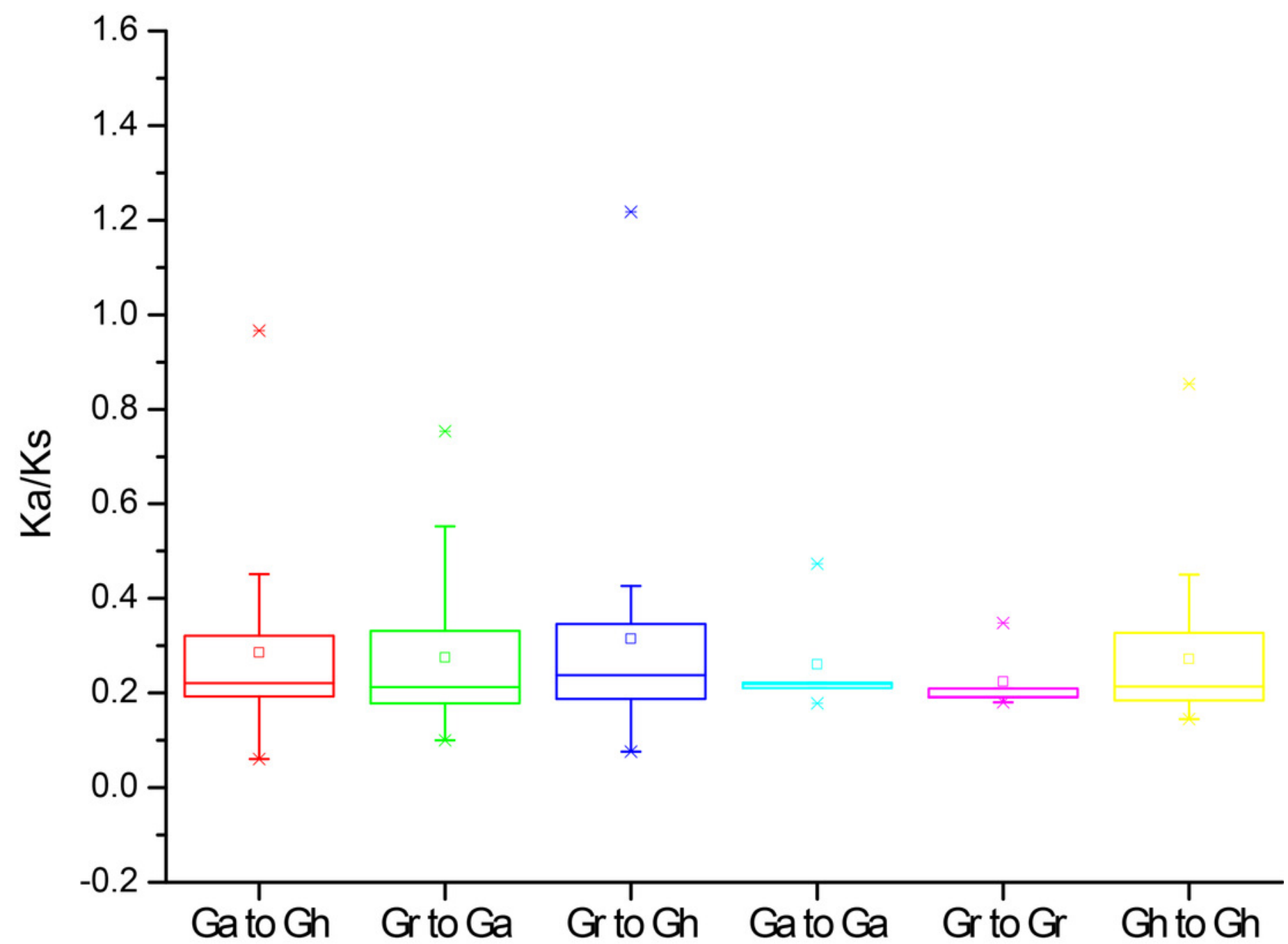


Figure 5

Phylogenetic tree of PP2CAs in Gossypium and other plants.

The multiple alignment was performed by MUSCLE program. IQTREE was used to create the maximum likelihood with Dayhoff model. The phylogenetic relationship of PP2CAs in G.

arboretum, G. raimondii, G. hirsutum, G. barbadense, A. thaliana, T. cacao, $R$. communis, $P$. trichocarpa, G. max, B. distachyon and O. sativa were analyzed.

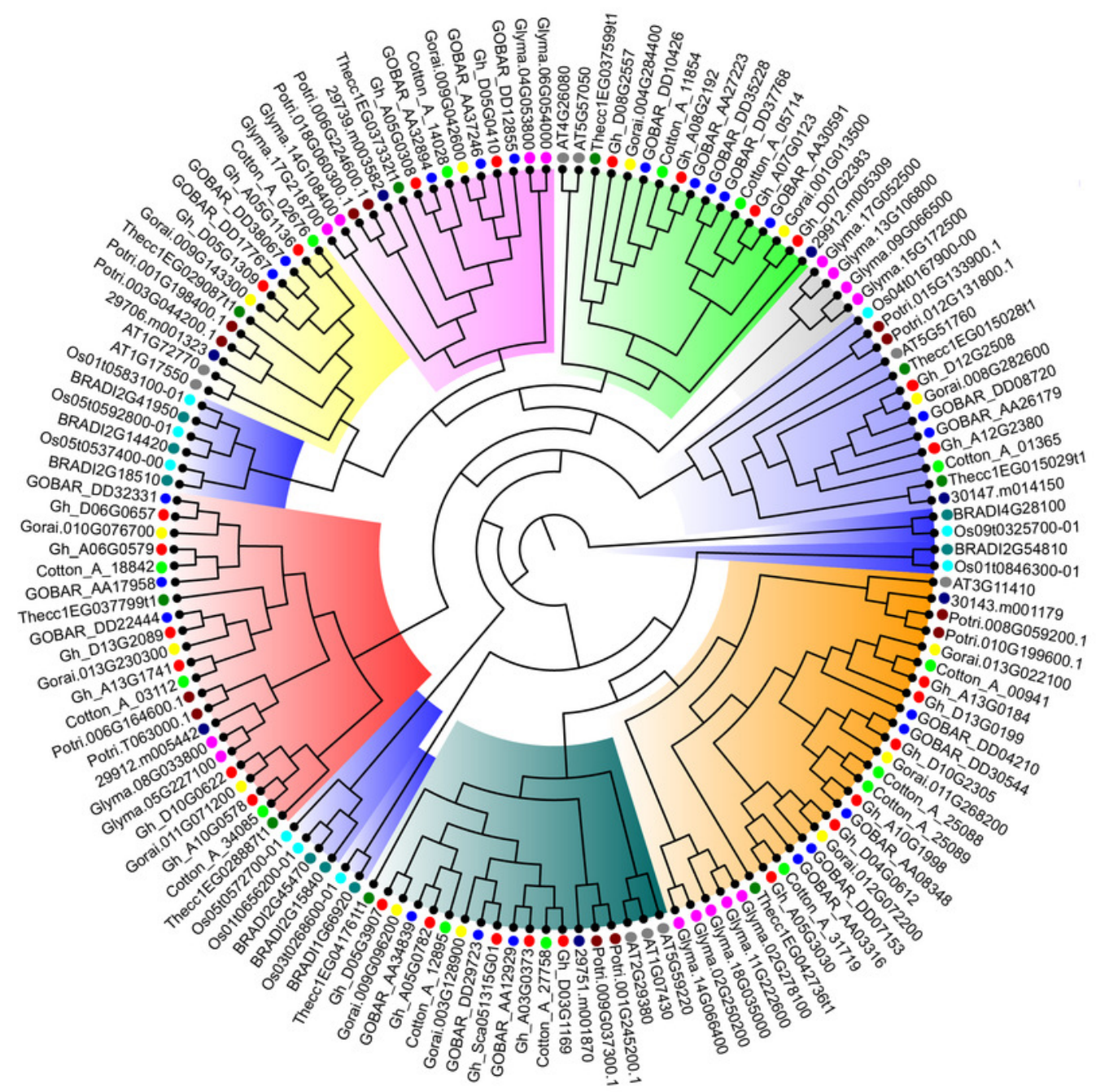

G. arboreum

G. raimondit

G. hirsutum

- G. barbadense

A. thaliana

- T. cacao

- R. Communis

- P trichocarpa

- G. max

- B. distachyon

O. sativa 


\section{Figure 6}

\section{Transcription levels of GhPP2CAs in cotton tissues.}

The genes preferentially expressed in flowers (A-Z) and fibers (AA). Gene GhUBQ7 was used as the internal control. The expression level of the gene in roots was set as 1 . The data were mean \pm SE. Statistical analyses were carried out by student's $t$-test to determine the differences in gene abundances between roots and other tissues. 

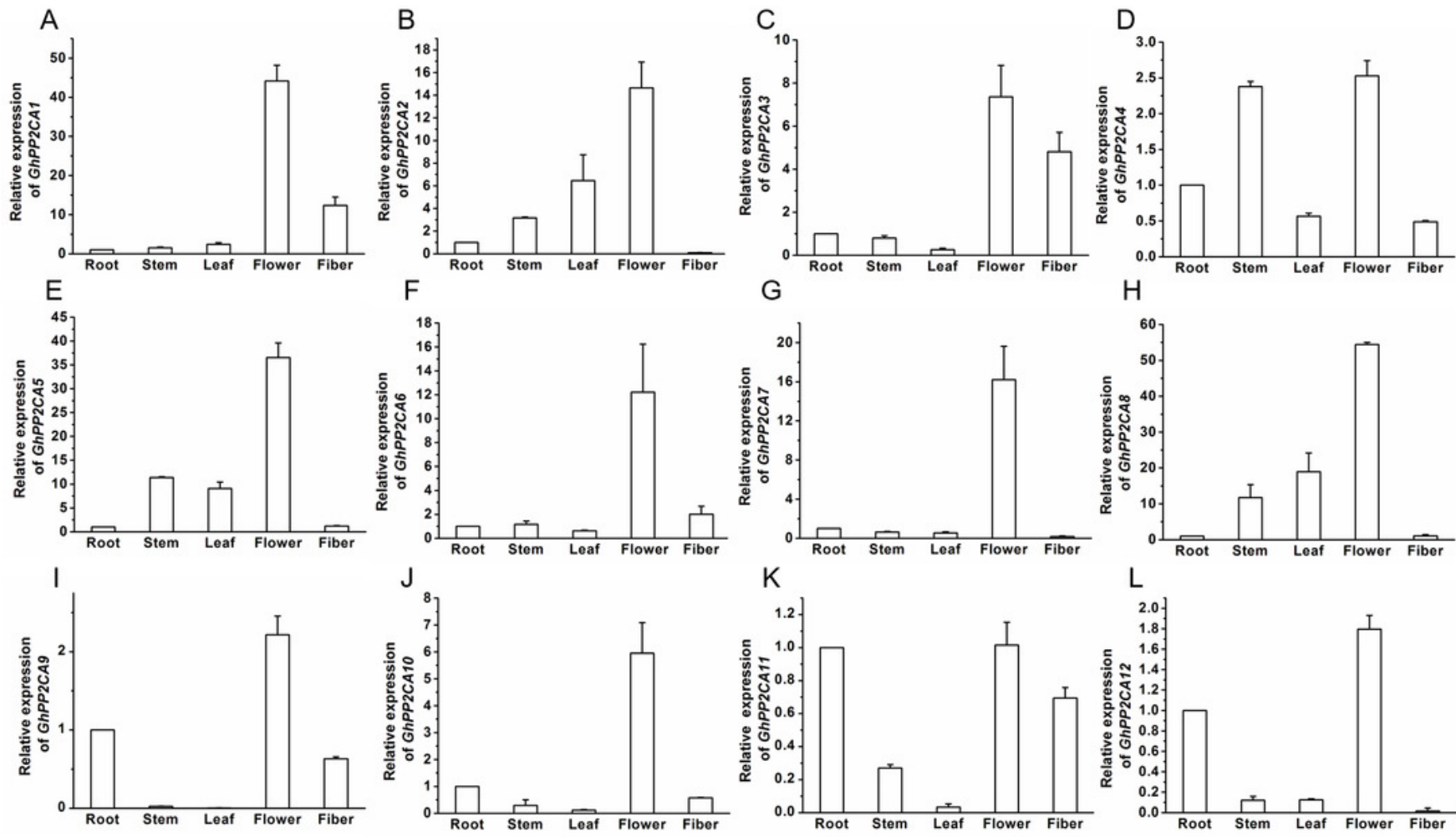

L
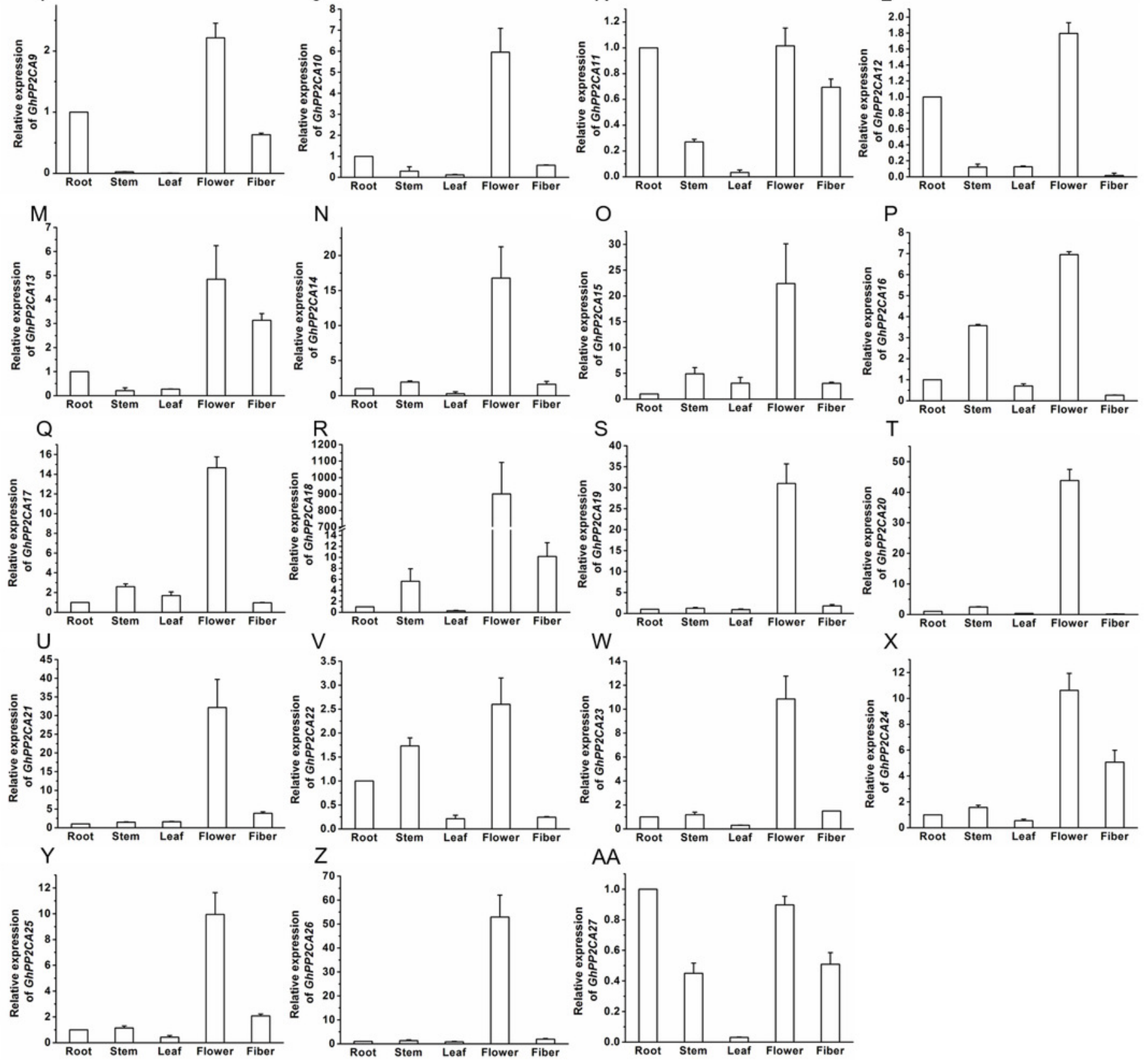

Peer) reviewing PDF | (2018:11:32799:2:0:NEW 2 May 2019) 


\section{Figure 7}

Expression of GhPP2CA genes in response to ABA.

The relative expression of GhPP2CAs was monitored after treatments with $100 \mu \mathrm{M}$ ABA for indicated period of time. The expression levels of the genes were prominently reduced at $3 \mathrm{~h}$ or $6 \mathrm{~h}$ but elevated at $12 \mathrm{~h}$ or $24 \mathrm{~h}(\mathrm{~A}-\mathrm{E})$, and were increased at $3 \mathrm{~h}$ or $6 \mathrm{~h}$ but decreased at $12 \mathrm{~h}$ or $24 \mathrm{~h}(\mathrm{~F}-\mathrm{O})$. The transcription abundances of the genes were generally enhanced ( $\mathrm{P}$ $\mathrm{U})$, and diminished (V-AA), respectively, with the prolongation of treatment time. Cotton gene GhUBQ7 was applied as the internal control. The gene expression value at $0 \mathrm{~h}$ was set as 1 . The values were mean $\pm S E$. Statistical analyses were carried out by student's $t$-test to determine the differences in gene abundances between $0 \mathrm{~h}$ and other treatment times. 

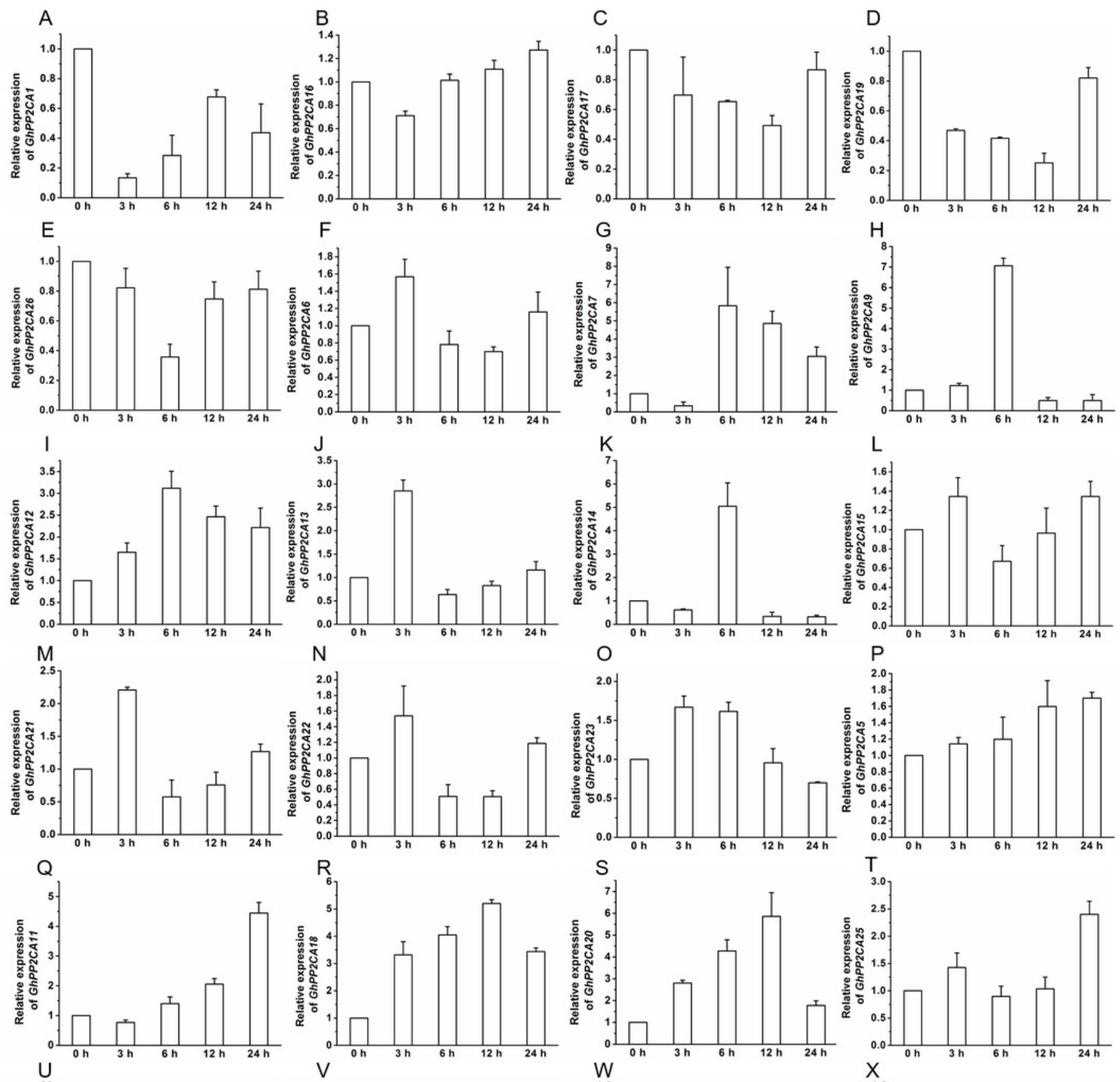

$\mathrm{T}$
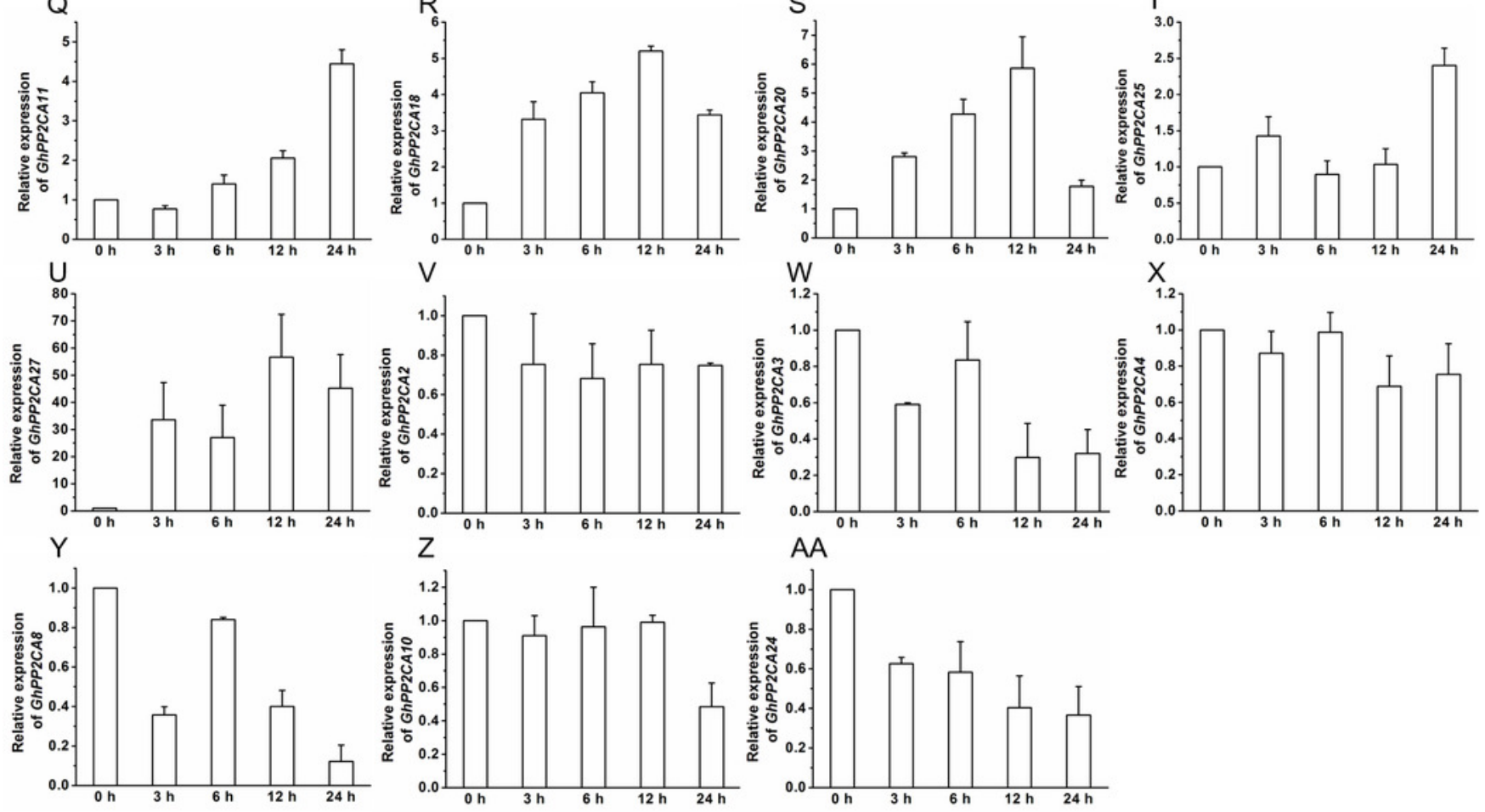


\section{Figure 8}

Expression of GhPP2CA genes in response to osmotic stress.

The relative expression of GhPP2CAs was determined after exposure upon 10\% PEG6000 for indicated time. The transcriptional levels of the genes were clearly decreased at $3 \mathrm{~h}$ or $6 \mathrm{~h}$ but increased at $12 \mathrm{~h}$ or $24 \mathrm{~h}(\mathrm{~A}-\mathrm{B})$, and rose at $3 \mathrm{~h}$ or $6 \mathrm{~h}$ but dropped down at $12 \mathrm{~h}$ or $24 \mathrm{~h}$ $(C-L)$, the expression of the genes in $(M-X)$ was elevated, and that in (Y-AA) was reduced with the extension of treatment time. GhUBQ7 was used as the internal control. The gene expression value at $0 \mathrm{~h}$ was set as 1 . The values were mean \pm SE. Statistical analyses were carried out by student's $t$-test to determine the differences in gene abundances between $0 \mathrm{~h}$ and other treatment times. 

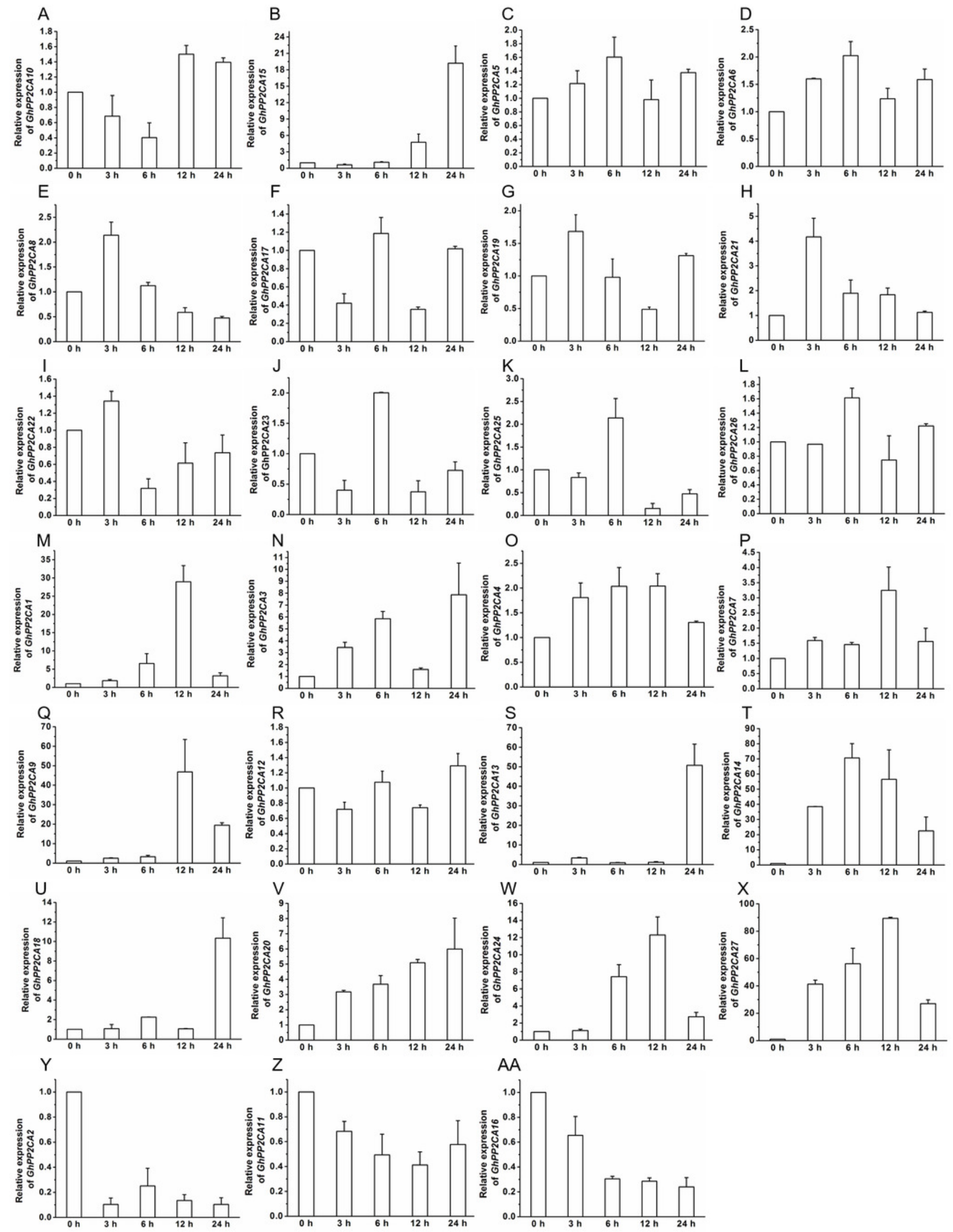


\section{Figure 9}

\section{Analysis of interactions between GhPP2CAs and GhPYL2-2D by yeast two-hybrid method.}

Narrowing triangles indicate the reduced cell densities in the dilution series from left to right.

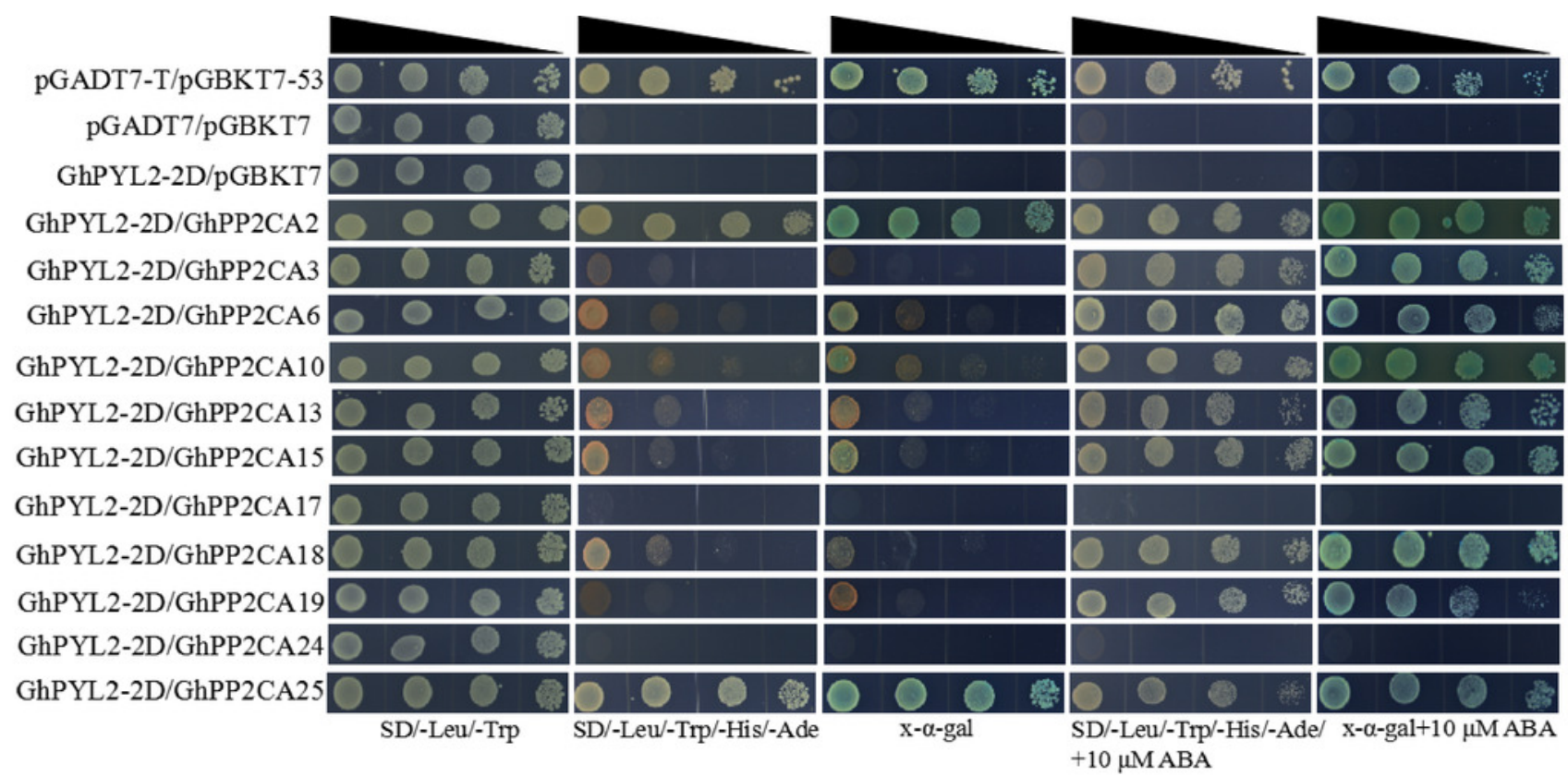




\section{Figure 10}

Assay of interactions between GhPP2CAs and GhPYL6-2A using yeast two-hybrid technology.

Narrowing triangles show the reduced cell densities in the dilution series from left to right.

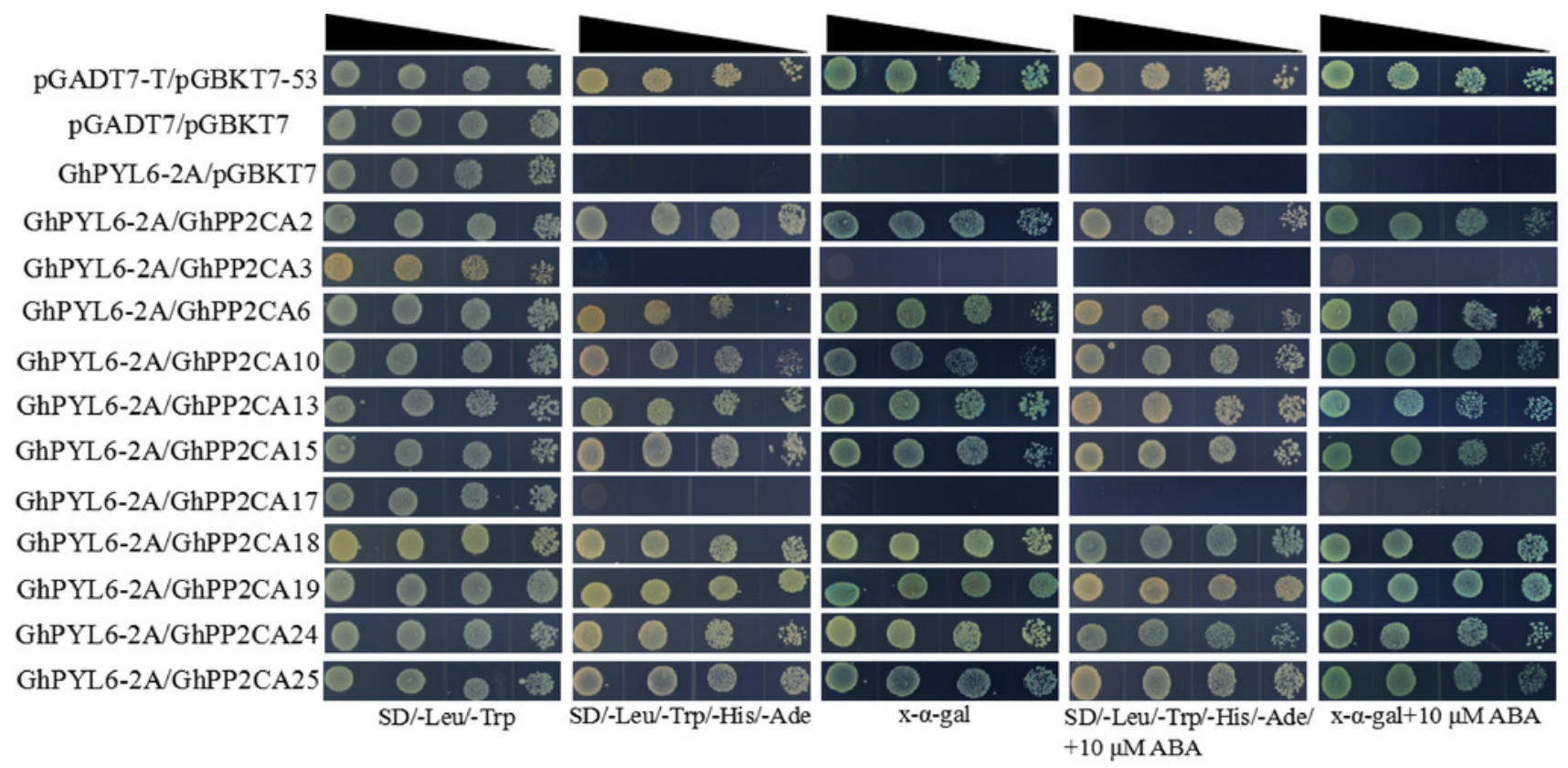




\section{Figure 11}

Determination of interactions between GhPP2CAs and GhPYL9-2A by yeast two-hybrid method.

Narrowing triangles reveal the reduced cell densities in the dilution series from left to right.

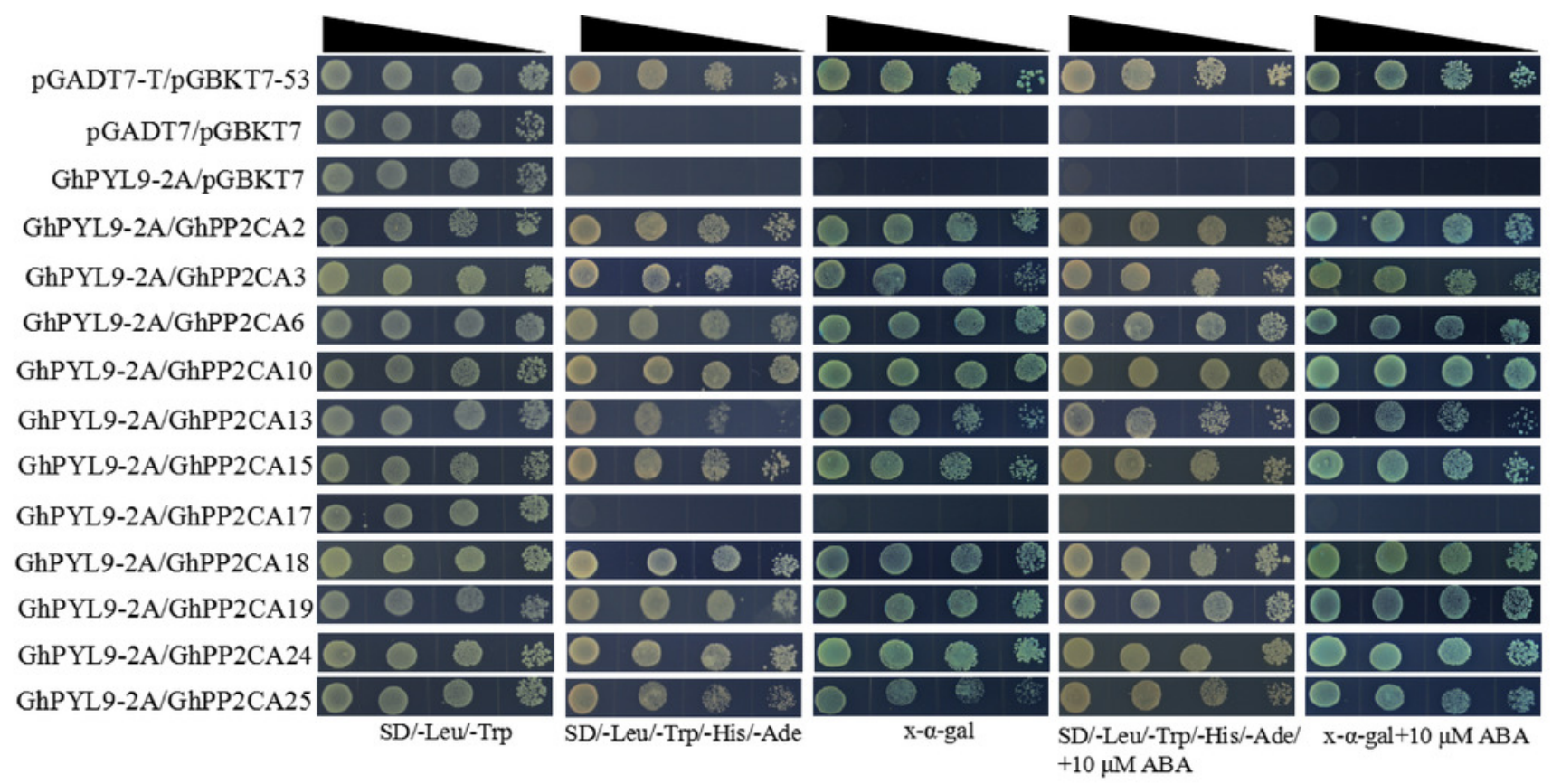


Table $\mathbf{1}$ (on next page)

PP2CA family genes in Gossypium. 


\begin{tabular}{|c|c|c|c|c|}
\hline Gene identifier & Gene name & $\begin{array}{l}\text { Size } \\
\text { (aa) }\end{array}$ & $\begin{array}{l}\text { Mass } \\
(\mathrm{kDa})\end{array}$ & $\mathrm{pI}$ \\
\hline Cotton_A_00941 & GaPP2CA1 & 420 & 46.9 & 6.61 \\
\hline Cotton_A_01365 & GaPP2CA2 & 419 & 45.9 & 6.39 \\
\hline Cotton_A_02676 & GaPP2CA3 & 494 & 53.4 & 5 \\
\hline Cotton_A_03112 & GaPP2CA4 & 400 & 43.4 & 5.12 \\
\hline Cotton_A_05714 & GaPP2CA5 & 470 & 51.8 & 5.82 \\
\hline Cotton_A_11854 & GaPP2CA6 & 593 & 66 & 5.51 \\
\hline Cotton_A_12895 & GaPP2CA7 & 416 & 45.4 & 6.07 \\
\hline Cotton_A_14028 & GaPP2CA8 & 573 & 62.4 & 4.69 \\
\hline Cotton_A_18842 & GaPP2CA9 & 393 & 43 & 5.25 \\
\hline Cotton_A_25088 & GaPP2CA10 & 413 & 45.1 & 5.15 \\
\hline Cotton_A_25089 & GaPP2CA11 & 413 & 45.1 & 5.15 \\
\hline Cotton_A_27758 & GaPP2CA12 & 390 & 43.3 & 8.34 \\
\hline Cotton_A_31719 & GaPP2CA13 & 413 & 45.4 & 5.55 \\
\hline Cotton_A_34085 & GaPP2CA14 & 242 & 26.6 & 5.73 \\
\hline Gh_A03G0373 & GhPP2CA1 & 416 & 45.9 & 8.09 \\
\hline Gh_A05G0308 & GhPP2CA2 & 558 & 60.9 & 4.69 \\
\hline Gh_A05G0782 & GhPP2CA3 & 416 & 45.4 & 6.07 \\
\hline Gh_A05G1136 & GhPP2CA4 & 494 & 53.5 & 5.09 \\
\hline Gh_A05G3030 & GhPP2CA5 & 413 & 45.4 & 5.52 \\
\hline Gh_A06G0579 & GhPP2CA6 & 416 & 45.9 & 5.64 \\
\hline Gh_A07G0123 & GhPP2CA7 & 471 & 52 & 5.58 \\
\hline Gh_A08G2192 & GhPP2CA8 & 463 & 51.4 & 7.56 \\
\hline Gh_A10G0578 & GhPP2CA9 & 397 & 42.8 & 5.76 \\
\hline Gh_A10G1998 & GhPP2CA10 & 409 & 44.6 & 5.1 \\
\hline Gh_A12G2380 & GhPP2CA11 & 420 & 46 & 6.27 \\
\hline Gh_A13G0184 & GhPP2CA12 & 179 & 20 & 5.71 \\
\hline Gh_A13G1741 & GhPP2CA13 & 400 & 43.3 & 5.12 \\
\hline Gh_D03G1169 & GhPP2CA14 & 258 & 28.1 & 8.6 \\
\hline Gh_D04G0612 & GhPP2CA15 & 413 & 45.5 & 5.61 \\
\hline Gh_D05G0410 & GhPP2CA16 & 558 & 60.8 & 4.69 \\
\hline Gh_D05G1309 & GhPP2CA17 & 494 & 53.5 & 5 \\
\hline Gh_D05G3907 & GhPP2CA18 & 417 & 45.4 & 5.83 \\
\hline Gh_D06G0657 & GhPP2CA19 & 393 & 43 & 5.49 \\
\hline Gh_D07G2383 & GhPP2CA20 & 471 & 51.9 & 5.91 \\
\hline Gh_D08G2557 & GhPP2CA21 & 463 & 51.3 & 6.48 \\
\hline Gh_D10G0622 & GhPP2CA22 & 342 & 38.1 & 8.74 \\
\hline Gh_D10G2305 & GhPP2CA23 & 411 & 45 & 5.05 \\
\hline Gh_D12G2508 & GhPP2CA24 & 418 & 45.8 & 6.42 \\
\hline Gh_D13G0199 & GhPP2CA25 & 416 & 46.2 & 6.53 \\
\hline
\end{tabular}




\begin{tabular}{|c|c|c|c|c|}
\hline Gh_D13G2089 & GhPP2CA26 & 400 & 43.4 & 5.17 \\
\hline Gh_Sca051315G01 & GhPP2CA27 & 118 & 12.8 & 4.93 \\
\hline GOBAR_AA03316 & GbPP2CA1 & 413 & 45.4 & 5.52 \\
\hline GOBAR_AA08348 & GbPP2CA2 & 409 & 44.6 & 5.1 \\
\hline GOBAR_AA12929 & GbPP2CA3 & 416 & 45.8 & 8.07 \\
\hline GOBAR_AA17958 & GbPP2CA4 & 393 & 43 & 5.31 \\
\hline GOBAR_AA26179 & GbPP2CA5 & 418 & 45.9 & 6.39 \\
\hline GOBAR_AA27223 & GbPP2CA6 & 456 & 50.6 & 7.16 \\
\hline GOBAR_AA30591 & GbPP2CA7 & 470 & 51.8 & 5.58 \\
\hline GOBAR_AA32894 & GbPP2CA8 & 558 & 60.9 & 4.67 \\
\hline GOBAR_AA34839 & GbPP2CA9 & 416 & 45.4 & 6.07 \\
\hline GOBAR_AA37246 & GbPP2CA10 & 558 & 60.8 & 4.69 \\
\hline GOBAR_DD04210 & GbPP2CA11 & 416 & 46.1 & 6.76 \\
\hline GOBAR_DD07153 & GbPP2CA12 & 371 & 40.7 & 6.07 \\
\hline GOBAR_DD08720 & GbPP2CA13 & 392 & 43.2 & 7.2 \\
\hline GOBAR_DD10426 & GbPP2CA14 & 385 & 42.4 & 6.64 \\
\hline GOBAR_DD12855 & GbPP2CA15 & 302 & 33.4 & 6.46 \\
\hline GOBAR_DD17767 & GbPP2CA16 & 389 & 42 & 4.96 \\
\hline GOBAR_DD22444 & GbPP2CA17 & 400 & 43.3 & 5.11 \\
\hline GOBAR_DD29723 & GbPP2CA18 & 408 & 44.9 & 8.52 \\
\hline GOBAR_DD30544 & GbPP2CA19 & 411 & 45 & 5.05 \\
\hline GOBAR_DD32331 & GbPP2CA20 & 226 & 25.2 & 5.58 \\
\hline GOBAR_DD35228 & GbPP2CA21 & 470 & 51.8 & 5.82 \\
\hline GOBAR_DD37768 & GbPP2CA22 & 470 & 51.8 & 5.82 \\
\hline GOBAR_DD38067 & GbPP2CA23 & 494 & 53.5 & 5 \\
\hline Gorai.001G013500 & GrPP2CA1 & 471 & 51.9 & 5.91 \\
\hline Gorai.003G128900 & GrPP2CA2 & 416 & 45.9 & 8.09 \\
\hline Gorai.004G284400 & GrPP2CA3 & 463 & 51.2 & 6.34 \\
\hline Gorai.008G282600 & GrPP2CA4 & 414 & 45.4 & 6.3 \\
\hline Gorai.009G042600 & GrPP2CA5 & 558 & 60.8 & 4.65 \\
\hline Gorai.009G096200 & GrPP2CA6 & 416 & 45.4 & 5.73 \\
\hline Gorai.009G143300 & GrPP2CA7 & 494 & 53.4 & 5.04 \\
\hline Gorai.010G076700 & GrPP2CA8 & 393 & 43 & 5.48 \\
\hline Gorai.011G071200 & GrPP2CA9 & 347 & 38.8 & 8.43 \\
\hline Gorai.011G268200 & GrPP2CA10 & 411 & 44.9 & 5 \\
\hline Gorai.012G072200 & GrPP2CA11 & 409 & 44.9 & 5.7 \\
\hline Gorai.013G022100 & GrPP2CA12 & 415 & 46.1 & 6.2 \\
\hline Gorai.013G230300 & GrPP2CA13 & 400 & 43.3 & 5 \\
\hline
\end{tabular}

1 